\title{
NWChem: Past, present, and future
}

Cite as: J. Chem. Phys. 152, 184102 (2020); https://doi.org/10.1063/5.0004997

Submitted: 17 February 2020 . Accepted: 07 April 2020 . Published Online: 11 May 2020

E. Aprà, (D) E. J. Bylaska, (D) W. A. de Jong, (D) N. Govind, (D) K. Kowalski, (iD) T. P. Straatsma, (D) M. Valiev, H. J. J. van Dam, (D) Y. Alexeev, J. Anchell, V. Anisimov, F. W. Aquino, R. Atta-Fynn, (D) J. Autschbach, (D) N. P. Bauman, J. C. Becca, (D) D. E. Bernholdt, K. Bhaskaran-Nair, S. Bogatko, P. Borowski, J. Boschen, J. Brabec, A. Bruner, E. Cauët, Y. Chen, (D) G. N. Chuev, (D) C. J. Cramer, J. Daily, M. J. O. Deegan, (D) T. H. Dunning, M. Dupuis, K. G. Dyall, G. I. Fann, S. A. Fischer, A. Fonari, H. Früchtl, (D) L. Gagliardi, J. Garza, N. Gawande, (iD S. Ghosh, K. Glaesemann, (D) A. W. Götz, (D) J. Hammond, (D) V. Helms, E. D. Hermes, K. Hirao, (D) S. Hirata, M. Jacquelin, (D) L. Jensen, (D) B. G. Johnson, (D) H. Jónsson, R. A. Kendall, (D) M. Klemm, (D) R. Kobayashi, V. Konkov, S. Krishnamoorthy, M. Krishnan, Z. Lin, R. D. Lins, R. J. Littlefield, (D) A. J. Logsdail, (D) K. Lopata, W. Ma, A. V. Marenich, (D) J. Martin del Campo, (D) D. Mejia-Rodriguez, J. E. Moore, J. M. Mullin, (D) T. Nakajima, D. R. Nascimento, J. A. Nichols, P. J. Nichols, J. Nieplocha, A. Otero-de-la-Roza, B. Palmer, (D) A. Panyala, (D) T. Pirojsirikul, (D) B. Peng, (D) R. Peverati, J. Pittner, L. Pollack, (D) R. M. Richard, P. Sadayappan, (D) G. C. Schatz, W. A. Shelton, D. W. Silverstein, D. M. A. Smith, (D) T. A. Soares, (D) D. Song, (D) M. Swart, H. L. Taylor, G. S. Thomas, V. Tipparaju, (D) D. G. Truhlar, K. Tsemekhman, T. Van Voorhis, Á. Vázquez-Mayagoitia, (D) P. Verma, O. Villa, A. Vishnu, K. D. Vogiatzis, (D) D. Wang, J. H. Weare, (D) M. J. Williamson, T. L. Windus, K. Woliński, A. T. Wong, (i) Q. Wu, C. Yang, Q. Yu, M. Zacharias, (D) Z. Zhang, (D. Zhao, and R. J. Harrison

\section{COLLECTIONS}

Paper published as part of the special topic on Electronic Structure SoftwareESS2020
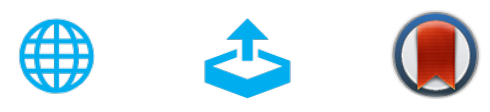

\section{ARTICLES YOU MAY BE INTERESTED IN}

PSI4 1.4: Open-source software for high-throughput quantum chemistry

The Journal of Chemical Physics 152, 184108 (2020); https://doi.org/10.1063/5.0006002

\section{TURBOMOLE: Modular program suite for ab initio quantum-chemical and condensed-} matter simulations

The Journal of Chemical Physics 152, 184107 (2020); https://doi.org/10.1063/5.0004635

Recent developments in the general atomic and molecular electronic structure system The Journal of Chemical Physics 152, 154102 (2020); https://doi.org/10.1063/5.0005188 


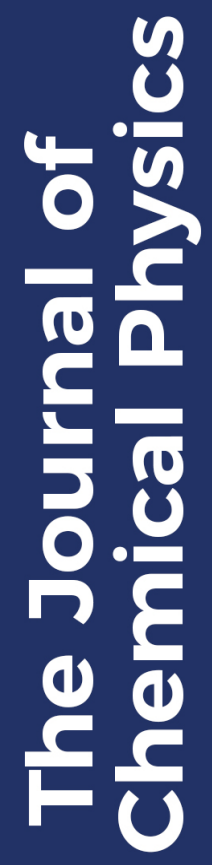

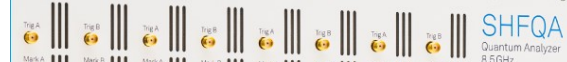

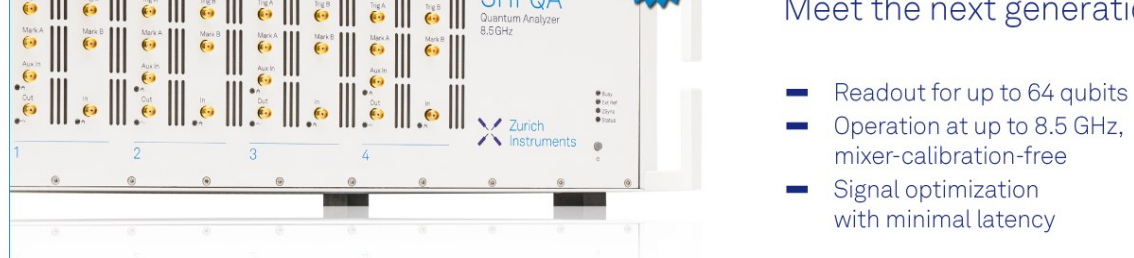

Your Qubits. Measured. Meet the next generation of quantum analyzers

- Readout for up to 64 qubits

peration at up to $8.5 \mathrm{GHz}$ with minimal latency

\section{Find out more}

Zurich

Instruments 


\section{NWChem: Past, present, and future}

Cite as: J. Chem. Phys. 152, 184102 (2020); doi: 10.1063/5.0004997

Submitted: 17 February 2020 - Accepted: 7 April 2020 •

Published Online: 11 May 2020

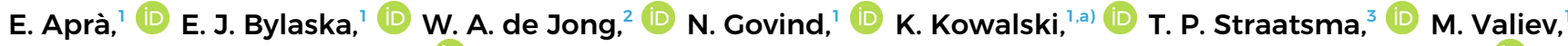
H. J. J. van Dam, ${ }^{4}$ Y. Alexeev, ${ }^{5}$ (D) J. Anchell, ${ }^{6}$ V. Anisimov, ${ }^{5}$ F. W. Aquino, ${ }^{7}$ R. Atta-Fynn, ${ }^{8}$ J. Autschbach, ${ }^{9}$ N. P. Bauman, ${ }^{1}$ (D) J. C. Becca, ${ }^{10}$ D. E. Bernholdt, ${ }^{11}$ (D) K. Bhaskaran-Nair, ${ }^{12}$ S. Bogatko, ${ }^{13}$ P. Borowski, ${ }^{14}$ J. Boschen, J. Brabec, ${ }^{16}$ A. Bruner, ${ }^{17}$ E. Cauët, ${ }^{18}$ Y. Chen, ${ }^{19}$ G. N. Chuev, ${ }^{20}$ (D) C. J. Cramer, ${ }^{21}$ (D) J. Daily, ${ }^{1}$ M. J. O. Deegan, ${ }^{22}$ T. H. Dunning, Jr., ${ }^{23}$ (D) M. Dupuis,, ${ }^{9}$ K. G. Dyall, ${ }^{24}$ G. I. Fann, ${ }^{17}$ S. A. Fischer, ${ }^{25}$ A. Fonari, ${ }^{26, b)}$ H. Früchtl, ${ }^{27}$ L. Gagliardi, ${ }^{21}$ (D) J. Garza, ${ }^{28}$ N. Gawande, ${ }^{1}$ S. Ghosh, ${ }^{29, c)}$ (D) K. Glaesemann, ${ }^{1}$ A. W. Götz, ${ }^{30}$ (D) J. Hammond, V. Helms, ${ }^{31}$ (D) E. D. Hermes, ${ }^{32}$ K. Hirao, ${ }^{33}$ S. Hirata, ${ }^{34}$ (D) M. Jacquelin, ${ }^{2}$ L. Jensen, ${ }^{10}$ (D) B. G. Johnson, ${ }^{35}$ H. Jónsson, ${ }^{36}$ (D) R. A. Kendall, ${ }^{17}$ M. Klemm, ${ }^{6}$ (D) R. Kobayashi, ${ }^{37}$ (D) V. Konkov, ${ }^{38}$ S. Krishnamoorthy, ${ }^{1}$ M. Krishnan, ${ }^{19}$ Z. Lin, ${ }^{39}$ R. D. Lins, ${ }^{40}$ R. J. Littlefield, ${ }^{41}$ A. J. Logsdail, ${ }^{42}$ (D) K. Lopata, ${ }^{43}$ (D) W. Ma, ${ }^{44}$ A. V. Marenich, ${ }^{45, d)}$

J. Martin del Campo, ${ }^{46}$ (D) D. Mejia-Rodriguez, ${ }^{47}$ (D) J. E. Moore, ${ }^{6}$ J. M. Mullin, ${ }^{48}$ T. Nakajima, ${ }^{49}$ (D) D. R. Nascimento,

J. A. Nichols, ${ }^{11}$ P. J. Nichols, ${ }^{50}$ J. Nieplocha, ${ }^{7}$ A. Otero-de-la-Roza, ${ }^{51}$ B. Palmer, ${ }^{1}$ A. Panyala, ${ }^{1}$ (D) T. Pirojsirikul, ${ }^{52}$

B. Peng, ${ }^{7}$ (D) Peverati, ${ }^{38}$ (D. Pittner, ${ }^{53}$ L. Pollack, ${ }^{54}$ R. M. Richard, ${ }^{55}$ (D) P. Sadayappan, ${ }^{56}$ G. C. Schatz,

W. A. Shelton, ${ }^{58}$ D. W. Silverstein, ${ }^{59}$ D. M. A. Smith, ${ }^{6}$ T. A. Soares, ${ }^{60}$ (D) D. Song, ${ }^{1}$ (D) M. Swart, ${ }^{61}$ (D) H. L. Taylor, ${ }^{62}$ G. S. Thomas, ${ }^{1}$ V. Tipparaju, ${ }^{63}$ D. G. Truhlar, ${ }^{21}$ (D) K. Tsemekhman, ${ }^{64}$ T. Van Voorhis, ${ }^{65}$ Á. Vázquez-Mayagoitia, ${ }^{5}$ P. Verma, ${ }^{66}$ (D) O. Villa, ${ }^{67}$ A. Vishnu, ${ }^{7}$ K. D. Vogiatzis, ${ }^{68}$ D. Wang, ${ }^{69}$ (D) J. H. Weare, ${ }^{70}$ M. J. Williamson, ${ }^{7}$ T. L. Windus, ${ }^{72}$ K. Woliński, ${ }^{14}$ A. T. Wong, ${ }^{73}$ Q. Wu, ${ }^{4}$ (D) Yang, ${ }^{2}$ Q. Yu, ${ }^{74}$ M. Zacharias, ${ }^{75}$ Z. Zhang, ${ }^{76}$ (D) Y. Zhao, ${ }^{77}$ (D) and R. J. Harrison ${ }^{78}$

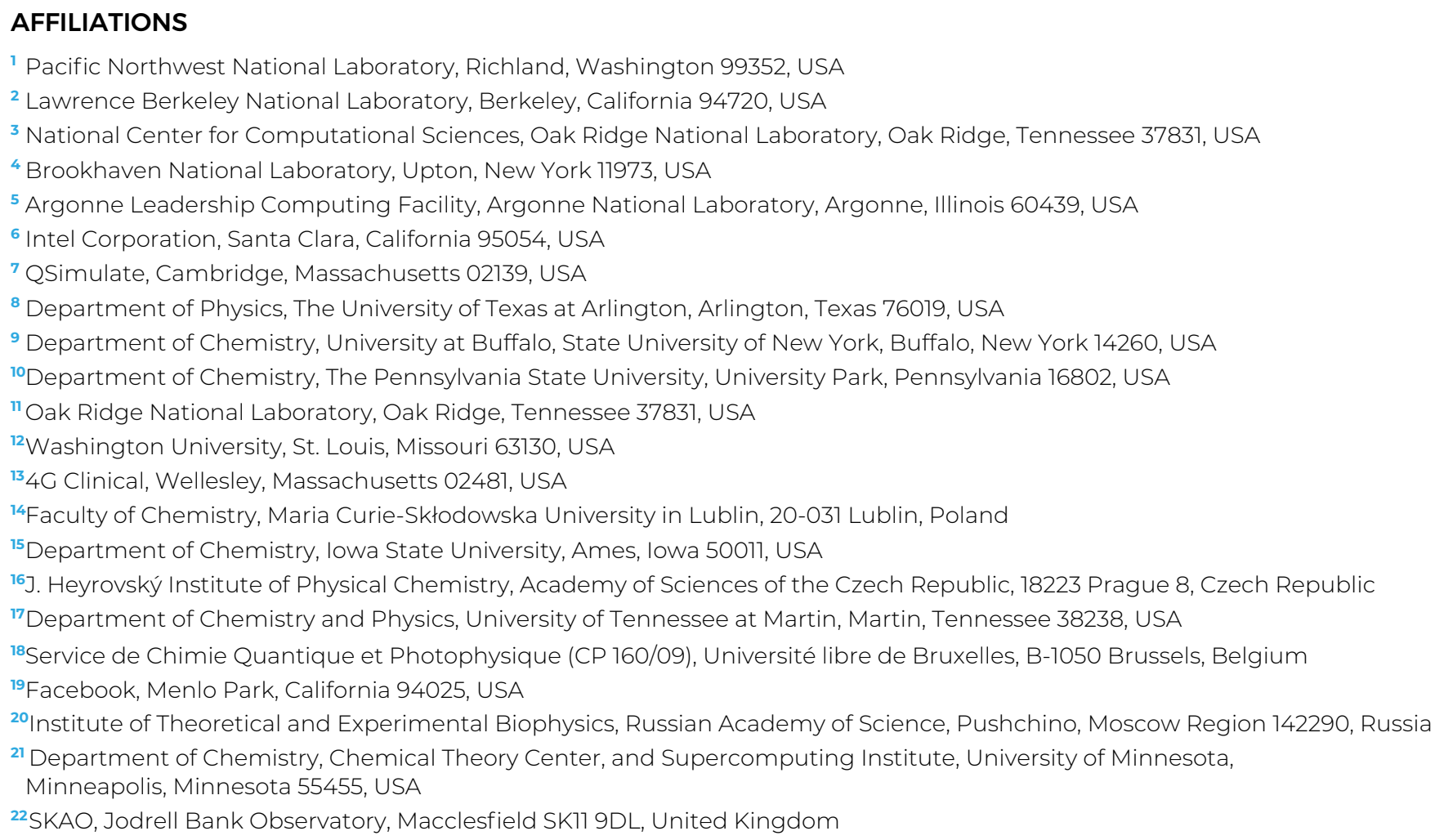


${ }^{23}$ Department of Chemistry, University of Washington, Seattle, Washington 98195, USA

${ }^{24}$ Dirac Solutions, Portland, Oregon 97229, USA

${ }^{25}$ Chemistry Division, U. S. Naval Research Laboratory, Washington, DC 20375, USA

${ }^{26}$ School of Chemistry and Biochemistry, Georgia Institute of Technology, Atlanta, Georgia 30332, USA

${ }^{27}$ EaStCHEM and School of Chemistry, University of St. Andrews, St. Andrews KY16 9ST, United Kingdom

${ }^{28}$ Departamento de Química, División de Ciencias Básicas e Ingeniería, Universidad Autónoma Metropolitana-Iztapalapa, Col. Vicentina, Iztapalapa, C.P. 09340 Ciudad de México, Mexico

${ }^{29}$ Department of Chemistry, Chemical Theory Center, and Supercomputing Institute, University of Minnesota, Minneapolis, Minnesota 5545, USA

${ }^{30}$ San Diego Supercomputer Center, University of California, San Diego, La Jolla, California 92093, USA

${ }^{31}$ Center for Bioinformatics, Saarland University, D-66041 Saarbrücken, Germany

${ }^{32}$ Combustion Research Facility, Sandia National Laboratories, Livermore, California 94551, USA

${ }^{33}$ Next-generation Molecular Theory Unit, Advanced Science Institute, RIKEN, Saitama 351-0198, Japan

${ }^{34}$ Department of Chemistry, University of Illinois at Urbana-Champaign, Urbana, Illinois 61801, USA

${ }^{35}$ Acrobatiq, Pittsburgh, Pennsylvania 15206, USA

${ }^{36}$ Faculty of Physical Sciences, University of Iceland, Reykjavík, Iceland and Department of Applied Physics, Aalto University, FI-00076 Aalto, Espoo, Finland

${ }^{37}$ ANU Supercomputer Facility, Australian National University, Canberra, Australia

${ }^{38}$ Chemistry Program, Florida Institute of Technology, Melbourne, Florida 32901, USA

${ }^{39}$ Department of Physics, University of Science and Technology of China, Hefei, China

${ }^{40} \mathrm{Aggeu}$ Magalhaes Institute, Oswaldo Cruz Foundation, Recife, Brazil

${ }^{41}$ Zerene Systems LLC, Richland, Washington 99354, USA

${ }^{42}$ Cardiff Catalysis Institute, School of Chemistry, Cardiff University, Cardiff, Wales CF10 3AT, United Kingdom

${ }^{43}$ Department of Chemistry, Louisiana State University, Baton Rouge, Louisiana 70803, USA

${ }_{44}$ Institute of Software, Chinese Academy of Sciences, Beijing, China

${ }^{45}$ Department of Chemistry, Chemical Theory Center, and Supercomputing Institute, University of Minnesota, Minneapolis, Minnesota 55455, USA

${ }^{46}$ Departamento de Física y Química Teórica, Facultad de Química, Universidad Nacional Autónoma de México, México City, Mexico

${ }^{47}$ Quantum Theory Project, Department of Physics, University of Florida, Gainesville, Florida 32611, USA

${ }^{48} \mathrm{DCl}$-Solutions, Aberdeen Proving Ground, Maryland 21005, USA

${ }^{49} \mathrm{C}$ mputational Molecular Science Research Team, RIKEN Center for Computational Science, Kobe, Hyogo 650-0047, Japan

${ }^{50}$ Los Alamos National Laboratory, Los Alamos, New Mexico 87545, USA

${ }^{51}$ Departamento de Química Física y Analítica, Facultad de Química, Universidad de Oviedo, 33006 Oviedo, Spain

${ }^{52}$ Department of Chemistry, Prince of Songkla University, Hat Yai, Songkhla 90112, Thailand

53J. Heyrovský Institute of Physical Chemistry, Academy of Sciences of the Czech Republic, v.v.i., 18223 Prague 8, Czech Republic

${ }^{54}$ StudyPoint, Boston, Massachusetts 02114, USA

${ }^{55}$ Ames Laboratory, Ames, lowa 50011, USA

${ }^{56}$ School of Computing, University of Utah, Salt Lake City, Utah 84112, USA

${ }^{57}$ Department of Chemistry, Northwestern University, Evanston, Illinois 60208, USA

${ }^{58}$ Cain Department of Chemical Engineering, Louisiana State University, Baton Rouge, Louisiana 70803, USA

${ }^{59}$ Universal Display Corporation, Ewing, New Jersey 08618, USA

${ }^{60}$ Dept. of Fundamental Chemistry, Universidade Federal de Pernambuco, Recife, Brazil

${ }^{61}$ ICREA, 08010 Barcelona, Spain and Universitat Girona, Institut de Química Computacional i Catàlisi, Campus Montilivi, 17003 Girona, Spain

${ }^{62}$ CD-adapco/Siemens, Melville, New York 11747, USA

${ }^{63}$ Cray Inc., Bloomington, Minnesota 55425, USA

${ }^{64}$ Gympass, New York, New York 10013, USA

${ }^{65}$ Department of Chemistry, Massachusetts Institute of Technology, Cambridge, Massachusetts 02139, USA

${ }^{66} 1 \mathrm{QBit}$, Vancouver, British Columbia V6E 4B1, Canada

${ }^{67}$ NVIDIA, Santa Clara, California 95051, USA 


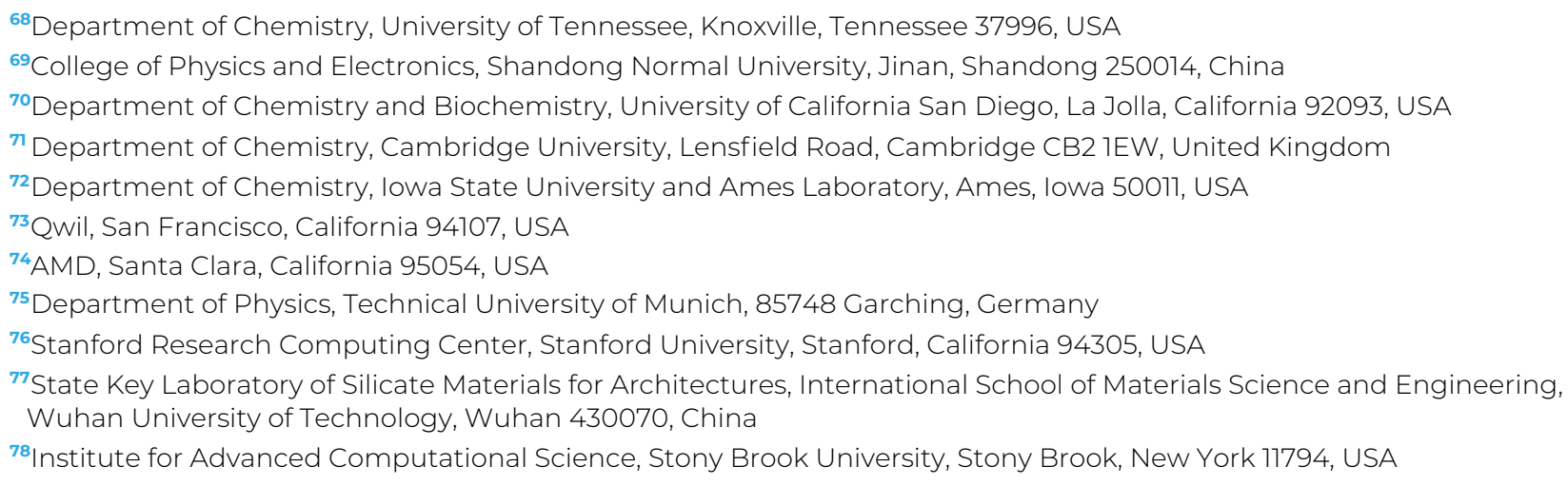

Note: This article is part of the JCP Special Topic on Electronic Structure Software.

a) Author to whom correspondence should be addressed: karol.kowalski@pnnl.gov

b) Current address: Schrödinger, Inc., New York, NY 10036, USA.

${ }^{c}$ Current address: Max Planck Institute für Kohlenforschung, 45470 Mülheim an der Ruhr, Germany.

d) Current address: Gaussian, Inc., Wallingford, CT 06492, USA.

\begin{abstract}
Specialized computational chemistry packages have permanently reshaped the landscape of chemical and materials science by providing tools to support and guide experimental efforts and for the prediction of atomistic and electronic properties. In this regard, electronic structure packages have played a special role by using first-principle-driven methodologies to model complex chemical and materials processes. Over the past few decades, the rapid development of computing technologies and the tremendous increase in computational power have offered a unique chance to study complex transformations using sophisticated and predictive many-body techniques that describe correlated behavior of electrons in molecular and condensed phase systems at different levels of theory. In enabling these simulations, novel parallel algorithms have been able to take advantage of computational resources to address the polynomial scaling of electronic structure methods. In this paper, we briefly review the NWChem computational chemistry suite, including its history, design principles, parallel tools, current capabilities, outreach, and outlook.
\end{abstract}

Published under license by AIP Publishing. https://doi.org/10.1063/5.0004997

\section{INTRODUCTION}

The NorthWest Chemistry (NWChem) modeling software is a popular computational chemistry package that has been designed and developed to work efficiently on massively parallel processing supercomputers. ${ }^{1-3}$ It contains an umbrella of modules that can be used to tackle most electronic structure theory calculations being carried out today. Since 2010, the code is distributed as open-source under the terms of the Educational Community License version 2.0 (ECL 2.0).

Electronic structure theory provides a foundation for our understanding of chemical transformations and processes in complex chemical environments. For this reason, accurate electronic structure formulations have already permeated several key areas of chemistry, biology, biochemistry, and materials sciences, where they have become indispensable elements for building synergies between theoretical and experimental efforts and for predictions. Over the past few decades, intense theoretical developments have resulted in a broad array of electronic structure methods and their implementations, designed to describe structures, interactions, chemical reactivity, dynamics, thermodynamics, and spectral properties of molecular and material systems. The success of these computational tools hinges upon several requirements regarding the accuracy of many-body models, reliable algorithms for dealing with processes at various spatial and temporal scales, and effective utilization of ever-growing computational resources. For instance, the predictive power of computational chemistry requires sophisticated quantum mechanical (QM) approaches that systematically account for electronic correlation effects. Therefore, the design of versatile electronic structure codes is a major undertaking that requires close collaboration between experts in theoretical and computational chemistry, applied mathematics, and computer science.

NWChem, ${ }^{2-8}$ like other widely used electronic structure programs, was developed to fully realize the potential of computational modeling to answer key scientific questions. It provides a wide range of capabilities that can be deployed on supercomputing platforms to solve two fundamental equations of quantum mechanics ${ }^{9-11}$ - timeindependent and time-dependent Schrödinger equations,

$$
\begin{gathered}
H|\Psi\rangle=E|\Psi\rangle, \\
i \hbar \frac{\partial|\Psi\rangle}{\partial t}=H|\Psi\rangle,
\end{gathered}
$$


and a fundamental equation of Newtonian mechanics,

$$
m_{i} \mathbf{a}_{i}=\mathbf{F}_{i},
$$

where forces $\mathbf{F}_{i}$ include information about quantum effects.

Given the breadth of electronic structure theory, it does not come as a surprise that Eqs. (1) and (2) can be solved using various representations of quantum mechanics employing wavefunctions $(|\Psi\rangle)$, electron densities $(\rho(\vec{r}))$, or self-energies $[\Sigma(\omega)]$, which comprise the wide spectrum of NWChem's functionalities to compute the electronic wavefunctions, densities, and associated properties of molecular and periodic systems. These functionalities include Hartree-Fock ${ }^{12-15}$ self-consistent field (SCF) and post-SCF correlated many-body approaches that build on the SCF wavefunction to tackle static and dynamic correlation effects. Among correlated approaches, NWChem offers second-order Möller-Plesset perturbation theory; single- and multi-reference (MR), groundand excited-state, and linear-response (LR) coupled-cluster (CC) theories; multi-configuration self-consistent field (MCSCF); and selected and full configuration interaction (CI) codes. NWChem provides extensive density functional theory ${ }^{16-18}$ (DFT) capabilities with Gaussian and plane wave basis set implementations. Within the Gaussian basis set framework, a broad range of DFT response properties, ground and excited-state molecular dynamics (MD), linear-response (LR), and real-time (RT) timedependent density functional theory (TDDFT) are available. The plane wave DFT implementations offer the capability to run scalable $a b$ initio and Car-Parrinello molecular dynamics ${ }^{19}$ and band structure simulations. The plane wave code supports both norm-conserving ${ }^{20-22}$ and projector augmented wave $(\mathrm{PAW})^{23}$ pseudopotentials.

For all DFT methods outlined above, both analytical or numerical gradients and Hessians are available for geometry optimization and vibrational analysis. Additionally, NWChem is capable of performing classical molecular dynamics (MD) simulations using either AMBER or CHARMM force fields. Through its modular design, the $a b$ initio methods can be coupled with the classical MD to perform mixed quantum-mechanics and molecular-mechanics simulations (QM/MM). Various solvent models and relativistic approaches are also available, with the spin-orbit (SO) contribution only being supported at the Hartree-Fock (HF) and DFT levels of theory and associated response properties. The NWChem functionality described is only a subset of its full capabilities. We refer the reader to the NWChem website 8 to learn about the full suite of functionalities available to the user community.

Currently, NWChem is developed and maintained primarily by researchers at the Department of Energy (DOE) Pacific Northwest National Laboratory (PNNL), with help from researchers at other research institutions. It has a broad user base, and it is being used across the national laboratory system and throughout academia and industry around the world. In this paper, we provide a high-level overview of NWChem's core capabilities, recent developments in electronic methods, and a short discussion of ongoing and future efforts. We also illustrate the strengths of NWChem stemming from the possibility of seamless integration of methodologies at various scales and review scientific results that would not otherwise be obtainable without using its highly scalable implementations of electronic structure methods.

\section{BRIEF HISTORY}

The NWChem project ${ }^{1-7,24,25}$ started in 1992. It was originally designed and implemented as part of the construction project associated with the EMSL user facility at PNNL. Therefore, the software project started around four years before the EMSL computing center was up and running. This raised challenges for the software developers working on the project, such as predicting the features of future hardware architectures and how to deliver high performing software while maintaining programmer productivity. Overcoming these challenges led to a design effort that strove for flexibility and extensibility, as well as high-level interfaces to functionality that hid some of the hardware issues from the chemistry software application developer. Over the years, this design and implementation have successfully advanced multiple science agendas, and NWChem's extensive code base of more than $2 \times 10^{6}$ lines provides high-performance, scalable software code with advanced scientific capabilities that are used throughout the molecular sciences community.

NWChem is an example of a co-design effort harnessing the expertise of researchers from multiple scientific disciplines to provide users with computational chemistry tools that are scalable both in their ability to treat large scientific computational chemistry problems efficiently and in their use of computing resources from highperformance parallel supercomputers to conventional workstation clusters. In particular, NWChem has been designed to handle

- biomolecules, nanostructures, interfaces, and solid-state,

- chemical processes in complex environments,

- hybrid quantum/classical simulations,

- ground and excited-states and non-linear optical properties,

- simulations of UV-Vis, photo-electron, and x-ray spectroscopies,

- Gaussian basis functions or plane waves,

- $a b$ initio molecular dynamics on the ground and excited states, and

- relativistic effects.

The scalability of NWChem has provided a computational platform to deliver new scientific results that would be unobtainable if parallel computational platforms were not used. For example, NWChem's implementation of a non-orthogonally spin adapted coupled-cluster single double triple $[\mathrm{CCSD}(\mathrm{T})]$ method has been demonstrated to scale to 210000 processors available at the Oak Ridge National Laboratory's (ORNL) Leadership Computing Facilities, ${ }^{26-28}$ whereas the plane wave DFT code has been able to utilize close to 100000 processor cores on NERSC's Cray-XE6 supercomputer. ${ }^{29}$ Although implemented only for the perturbative part of coupled-cluster with singles and doubles (CCSD) $)^{30}$ and triples correction $[\operatorname{CCSD}(\mathrm{T})],{ }^{31} \mathrm{NWChem}$ was one of the first computational chemistry codes to have been ported to utilize graphics processing units (GPUs). ${ }^{32}$ Several parts of the code have also been rewritten to take advantage of the Intel Xeon Phi family of processorsgood scalability and performance have been demonstrated for the ab initio molecular dynamics (AIMD) plane wave DFT code on the most recent Knights Landing version of the processor. ${ }^{33,34}$ The noniterative triples part of the $\operatorname{CCSD}(\mathrm{T})$ method has been demonstrated to scale to 55200 Intel Phi threads and 62560 cores through concurrent utilization of central processing unit (CPU) and Intel Xeon Phi Knights Corner accelerators. 


\section{DESIGN PRINCIPLES}

NWChem has a five-tiered modular architecture. The first tier is the Generic Task Interface. This interface (an abstract programming interface, not a user interface) serves as the mechanism that transfers control to the different modules in the second tier, which consists of the Molecular Calculation Modules. The molecular calculation modules are the high-level programming modules that accomplish computational tasks, performing particular operations using the specified theories defined by the user in the input file. These independent modules of NWChem share data only through a disk-resident database, which allows modules to share data or to share access to files containing data. The third tier consists of the Molecular Modeling Tools. These routines provide basic chemical functionality such as symmetry, basis sets, grids, geometry, and integrals. The fourth tier is the Software Development Toolkit, which is the basic foundation of the code. The fifth tier provides the Utility Functions needed by nearly all modules in the code. These include functionality such as input processing, output processing, and timing.

The Generic Task Interface controls the execution of NWChem. The flow of control proceeds in the following steps:

1. Identify and open the input file.

2. Complete the initialization of the parallel environment.

3. Process start-up directives.

4. Summarize start-up information and write it to the output file.

5. Open the run-time database.

6. Process the input sequentially (ignoring start-up directives), including the first task directive.

7. Execute the task.

8. Repeat steps 6 and 7 until reaching the end of the input file or encountering a fatal error condition.

The input parser processes the user's input file and translates the information into a form meaningful to the main program and the driver routines for specific tasks.

As mentioned in step 5 of the task flow control, NWChem makes use of a run-time database to store the main computational parameters. This is in the same spirit of check-pointing features available in other quantum chemistry codes. The information stored in the run-time database can be used at a later time in order to restart a calculation. Restart capabilities are available for most modules. For example, SCF generated files (run-time database and molecular orbitals) can be used either to continue a geometry optimization or to compute molecular properties. The important second and fourth tiers are discussed as part of Secs. IV-VI.

\section{PARALLEL TOOLS}

The design and early development of the Global Arrays ${ }^{36-39}$ (GA) toolkit occurred in the same period when the NWChem project started. The GA toolkit, which is the central component of the Software Development Toolkit, was adopted by the NWChem developers as the main approach for the parallelization of the dense matrices present in quantum chemistry methods that make use of local basis functions. In current computer science parlance, global arrays can be viewed as a Partitioned Global Address Space (PGAS) model that provides a high level of abstraction for the programmer to the dense distributed arrays. In contrast to message passing constructs such as Message Passing Interface (MPI), where the developer has to worry about coordinating send and receive operations, the use of global arrays in NWChem requires the so-called singlesided functions (e.g., put, get, and accumulate) to manipulate data structures in a single operation. The choice of the distribution model for sharing a given global array among the memory available to the processes in use plays a crucial role in efficient parallelization at large scale.

The GA toolkit has been ported to a variety of parallel computer architectures. The porting process has focused in the past in optimizing the ARMCI ${ }^{40}$ library. The Aggregate Remote Memory Copy (ARMCI) library optimizes performance by fully exploiting network characteristics such as latency, bandwidth, and packet injection rate through the use of low-level network protocols (e.g., Infiniband verbs). More recent porting options make use of either ComEx ${ }^{41}$ or the ARMCI-MPI ${ }^{42}$ communication runtimes. Both ComEx and ARMCI-MPI make use of MPI libraries, instead of low-level network protocols, albeit with different approaches.

\section{MAIN METHODOLOGIES}

In this section, we describe the key methods that comprise the Molecular Calculation Modules. We first describe the Gaussian basis HF and DFT implementations for molecular systems. This is followed by the post-SCF wavefunction-based perturbative (MP2), multi-configuration SCF, and high accuracy (coupledcluster theory) approaches for molecules, including the tensor contraction engine (TCE). Molecular response properties and relativistic approaches are then described. The plane wave based DFT implementation for Car-Parrinello molecular dynamics and periodic condensed phase systems is described next, followed by classical molecular dynamics and hybrid methods.

\section{A. Hartree-Fock}

The NWChem SCF module computes closed-shell restricted Hartree-Fock (RHF) wavefunctions, restricted high-spin openshell Hartree-Fock (ROHF) wavefunctions, and spin-unrestricted Hartree-Fock (UHF) wavefunctions. The Hartree-Fock equations are solved using a conjugate-gradient method with an orbital Hessian based preconditioner.

The most expensive part to compute in the SCF code is the two-electron contribution to the matrix element of the Fock operator (resulting from the sum of Coulomb and exchange operators). To compute these matrix elements, NWChem developers have implemented parallel algorithms using either a distributed data approach $^{44}$ (where the Fock matrix is distributed among the aggregate memory of the processes involved in the calculation) or a replicated data approach (where an entire copy of the Fock matrix is stored in the memory of each process).

Several options are available for the initial guess of the SCF calculations. The default choice uses the eigenvectors of a Focklike matrix formed from a superposition of the atomic densities. Other options include the use of eigenvectors of the bare-nucleus Hamiltonian or the one-electron Hamiltonian, the projections of the molecular orbital from a smaller basis to a larger one, or molecular orbitals formed by superimposing the orbitals of fragments of the 
molecule being studied. Symmetry can be used to speed up the Fock matrix construction via the petite-list algorithm. Molecular orbitals are symmetry adapted as well in NWChem. The resolution of the identity (RI) four-center, two-electron integral approximation has also been implemented.

In order to avoid full matrix diagonalization, the SCF program uses a preconditioned conjugate gradient (PCG) method that is unconditionally convergent. Basically, a search direction is generated by multiplying the orbital gradient (the derivative of the energy with respect to the orbital rotations) by an approximation to the inverse of the level-shifted orbital Hessian. In the initial iterations, an inexpensive one-electron approximation to the inverse orbital Hessian is used. Closer to convergence, the full orbital Hessian is used, which should provide quadratic convergence. For both the full or one-electron orbital Hessians, the inverse-Hessian matrixvector product is formed iteratively. Subsequently, an approximate line search is performed along the new search direction.

Both all-electron basis sets and effective core potentials (ECPs) can be used. Effective core potentials are a useful means of replacing the core electrons in a calculation with an effective potential, thereby eliminating the need for the core basis functions, which usually require a large set of Gaussians to describe them. In addition to replacing the core, they may be used to represent relativistic effects, which will be discussed later.

\section{B. Density functional theory}

The NWChem DFT module for molecular systems uses a Gaussian basis set to compute closed- and open-shell densities and Kohn-Sham orbitals in the local density approximation (LDA), generalized gradient approximation (GGA), $\tau$-dependent and Laplacian-dependent meta-generalized gradient approximation (meta-GGA), any combination of local and non-local approximations (including exact exchange and range-separated exchange), and asymptotically corrected exchange-correlation potentials. NWChem contains energy-gradient implementations of most exchangecorrelation functionals available in the literature, including a flexible framework to combine different functionals. However, second derivatives are not supported for meta-functionals, and third derivatives are supported only for a selected set of functionals. For a detailed description, we refer the reader to the online documentation. ${ }^{4}$

The DFT module reuses elements of the Gaussian basis SCF module for the evaluation of the Hartree-Fock exchange and the Coulomb matrices by using 4-index 2-electron electron repulsion integrals; the formal scaling of the DFT computation can be reduced by choosing to use auxiliary Gaussian basis sets to fit the charge density ${ }^{47}$ and use 3-index 2-electron integrals instead.

The DFT module supports both the distributed data approach and the mirrored array ${ }^{48}$ approach for the evaluation of the exchange-correlation potential and energy. The mirrored array option, used by default, allows the calculation to hide network communication overhead by replicating the data between processes belonging to the same network node.

In analogy with what is available in the SCF module, the DFT module can perform restricted closed-shell, unrestricted open-shell, and restricted open-shell calculations. However, in contrast to the SCF module that uses PCG to solve the SCF equation, the DFT module implements diagonalization with parallel eigensolvers. ${ }^{49-54}$ DIIS (direct inversion in the iterative subspace or direct inversion of the iterative subspace), ${ }^{55}$ level-shifting, ${ }^{56,57}$ and density matrix damping can be used to accelerate the convergence of the iterative SCF process. Another technique that can be used to help SCF convergence makes use of electronic smearing of the molecular orbital occupations by using a gaussian broadening function following the prescription of Warren and Dunlap. ${ }^{58}$ Additionally, calculations with fractional numbers of electrons can be performed to analyze the behavior of exchange-correlation functionals and their impact on molecular excited states and response properties. ${ }^{59-66}$

The Perdew and Zunger ${ }^{67}$ method to remove the selfinteraction contained in many exchange-correlation functionals has been implemented ${ }^{68}$ within the Optimized Effective Potential (OEP) method ${ }^{69,70}$ and within the Krieger-Li-Iafrate (KLI) approximation. $^{71,72}$

The asymptotic region of the exchange-correlation potential can be modified by the van-Leeuwen-Baerends exchangecorrelation potential that has the correct $-\frac{1}{r}$ asymptotic behavior. The total energy is then computed using the definition of the exchange-correlation functional. This scheme is known to tend to over-correct the deficiency of most uncorrected exchangecorrelation potentials ${ }^{73,74}$ and can improve TDDFT-based excitation calculations, but it is not variational. A variationally consistent approach to address this issue is via range-separated exchange-correlation functionals and the recently developed nearly correct asymptotic potential or NCAP, ${ }^{75}$ which are implemented in NWChem.

To describe dispersion interactions, both the exchange-hole dipole moment (XDM) dispersion model ${ }^{76}$ and Grimme's DFT-D3 dispersion correction (both zero-damped and BJ-damped variants) for DFT functionals ${ }^{77,78}$ are available. In many cases, one can obtain reasonably accurate non-covalent interaction energies at van der Waals distances with meta-functionals in NWChem even without adding extra dispersion terms.

Numerical integration is necessary for the evaluation of the exchange-correlation contribution to the density functional when Gaussian basis functions are used. The three-dimensional molecular integration problem is reduced to a sum of atomic integrations by using the approach first proposed by Becke. ${ }^{80}$ NWChem implements a modification of the Stratmann algorithm, ${ }^{81}$ where the polynomial partition function $w_{A}(r)$ is replaced by a modified error function erf $n$ (where $n$ can be 1 or 2),

$$
\begin{aligned}
w_{A}(r) & =\prod_{B \neq A} \frac{1}{2}\left[1-\operatorname{erf}\left(\mu_{A B}^{\prime}\right)\right], \\
\mu_{A B}^{\prime} & =\frac{1}{\alpha} \frac{\mu_{A B}}{\left(1-\mu_{A B}^{2}\right)^{n}}, \\
\mu_{A B} & =\frac{\mathbf{r}_{A}-\mathbf{r}_{B}}{\left|\mathbf{r}_{A}-\mathbf{r}_{B}\right|} .
\end{aligned}
$$

The default quadrature used for the atomic centered numerical integration is an Euler-MacLaurin scheme for the radial components (with a modified Mura-Knowles ${ }^{82}$ transformation) and a Lebedev $^{83}$ scheme for the angular components.

On top of the petite-list symmetry algorithm used in the same fashion as in the SCF module, the evaluation of the exchangecorrelation kernel incurs additional time savings when the molecular 
symmetry is a subset of the $O_{h}$ point group, exploiting the octahedral symmetry of the Lebedev angular grid.

NWChem also has an implementation of a variational treatment of the one-electron spin-orbit operator within the DFT framework. Calculations can be performed either with an all-electron relativistic approach [for example, zeroth order regular approximation (ZORA)] or with an ECP and a matching spin-orbit (SO) potential.

Other capabilities built on the DFT module include the electron transfer (ET), ${ }^{84,85}$ constrained DFT (CDFT), ${ }^{86-88}$ and frozen density embedding (FDE) ${ }^{89-91}$ modules, respectively.

\section{Time-dependent density functional theory}

a. Linear-response time-dependent density functional theory. NWChem supports a spectrum of single excitation theories for vertical excitation energy calculations, namely, configuration interaction singles (CIS), ${ }^{92}$ time-dependent Hartree-Fock [TDHF or also known as random-phase approximation (RPA)], timedependent density functional theory (TDDFT), ${ }^{93-95}$ and TammDancoff approximation $^{96}$ to TDDFT. These methods are implemented in a single framework that invokes Davidson's trial vector algorithm (or its modification for a non-Hermitian eigenvalue problem). An efficient special symmetric Lanczos algorithm and a kernel polynomial method have also been implemented.

In addition to valence vertical excitation energies, core-level excitations ${ }^{98}$ and emission spectra ${ }^{99,100}$ can also be computed. Analytical first derivatives of vertical excitation energies with a selected set of exchange-correlation functionals can also be computed, ${ }^{101}$ which allows excited-state optimizations and dynamics. Originindependent optical rotation and rotatory strength tensors can also be calculated with the LR-TDDFT module within the gauge including atomic orbital (GIAO) basis formulation. ${ }^{62,102-104}$ Extensions to compute excited-state couplings are currently underway and will be available in a future release.

b. Real-time time-dependent density functional theory. Realtime time-dependent density functional theory (RT-TDDFT) is a DFT-based approach to electronic excited states based on integrating the time-dependent Kohn-Sham (TDKS) equations in time. The theoretical underpinnings, strengths, and limitations are similar to traditional linear-response (LR) TDDFT methods, but instead of a frequency domain solution to the TDKS equations, RT-TDDFT yields a full time-resolved, potentially non-linear solution. Real-time simulations can be used to compute not only spectroscopic properties (e.g., ground and excited-state absorption spectra and polarizabilities) ${ }^{98,105-108}$ but also the time and space-resolved electronic response to arbitrary external stimuli (e.g., electron charge dynamics after laser excitation) ${ }^{105,109}$ and non-linear spectroscopies. ${ }^{110,111}$ RT-TDDFT has the potential to be efficient for computing spectra in systems with a high density of states ${ }^{112}$ as, in principle, an entire absorption spectrum can be computed from only one dynamics simulation.

This functionality is developed on the Gaussian basis set DFT module for both restricted and unrestricted calculations and can be run with essentially any combination of basis set and exchangecorrelation functional in NWChem. A number of time propagation algorithms have been implemented ${ }^{113}$ within this module, with the default being the Magnus propagator. ${ }^{114}$ Unlike LR-TDDFT, which requires second derivatives, RT-TDDFT can be used with all the functionals since only first derivatives are needed for the propagation. The current RT-TDDFT implementation assumes frozen nuclei and no dissipation.

\section{Ab initio molecular dynamics}

This module leverages the Gaussian basis set methods to allow for seamless molecular dynamics of molecular systems. The nuclei are treated as classical point particles, and their motion is integrated via the velocity Verlet algorithm. ${ }^{115,116}$ In addition to being able to perform simulations in the microcanonical ensemble, we have implemented several thermostats to control the kinetic energy of the nuclei. These include the stochastic velocity rescaling approach of Bussi, Donadio, and Parrinello, ${ }^{117}$ Langevin dynamics according to the implementation of Bussi and Parrinello, ${ }^{118}$ the Berendsen thermostat, ${ }^{119}$ and simple velocity rescaling.

The potential energy surface upon which the nuclei move can be provided by any level of theory implemented within NWChem, including DFT, TDDFT, MP2, and the correlated wavefunction methods in the TCE module. If analytical gradients are implemented for the specified method, these are automatically used. Numerical gradients will be used in the event that analytical gradients are not available at the requested level of theory. This module has been used to demonstrate how the molecular dynamics based determination of vibrational properties can complement those determined through normal mode analysis, therefore allowing to achieve a deeper understanding of complex dynamics and to help interpret complex experimental signatures. ${ }^{120}$ Extensions to include non-adiabatic dynamics have been implemented in a development version and will be available in a future release.

\section{Wavefunction formulations}

The wavefunction-based methods play a special role in all electronic structure packages. Their strengths originate in the possibility of introducing, using either various orders of perturbation theory or equivalently through the linked cluster theorem (see Refs. 121 and 122) various ranks of excitations, a systematic hierarchy of electron correlation effects. NWChem offers implementations of several correlated wavefunction approaches, including many-body perturbation theory (MBPT) approaches and coupled-cluster methods.

\section{Perturbative formulations}

a. MP2. Three algorithms are available in NWChem to compute the Møller-Plesset (or many-body) perturbation theory second-order correction ${ }^{123}$ to the Hartree-Fock energy (MP2). They vary in capability, the size of the system that can be treated, and the use of other approximations:

- Semi-direct MP2 is recommended for most large applications on parallel computers with significant disk I/O capability. Partially transformed integrals are stored on disk, multi-passing as necessary. RHF and UHF references may be treated including the computation of analytic derivatives. The initial semi-direct code was later modified to use aggregate memory instead of disk to store intermediate, therefore not requiring any $\mathrm{I} / \mathrm{O}$ operation. 
- Fully direct ${ }^{124}$ MP2. This is of use if only limited I/O resources are available (up to about 2800 functions). Only RHF references and energies are available.

- The resolution of the identity (RI) approximation MP2 (RIMP2) ${ }^{125}$ uses the RI approximation and is, therefore, only exact in the limit of a complete fitting basis. However, with some care, high accuracy may be obtained with relatively modest fitting basis sets. An RI-MP2 calculation can cost over 40 times less than the corresponding exact MP2 calculation. RHF and UHF references with only energies are available.

\section{Multi-configurational self-consistent field (MCSCF)}

A large-scale parallel multi-configurational self-consistent field (MCSCF) method has been developed in NWChem by the integration of the serial LUCIA program of Olsen. ${ }^{126,127}$ The generalized active space approach is used to partition large configuration interaction $(\mathrm{CI})$ vectors and generate a sufficient number of nearly equal batches for parallel distribution. This implementation allows the execution of complete active space self-consistent field (CASSCF) calculations with non-conventional active spaces. An unprecedented CI step for an expansion composed of almost one trillion Slater determinants has been reported.

\section{Coupled-cluster theory}

The coupled-cluster module of NWChem contains two classes of implementations: (a) parallel implementation of the $\operatorname{CCSD}(\mathrm{T})$ formalism $^{31}$ for closed-shell systems and (b) a wide array of CC formalisms for arbitrary reference functions. The latter class of implementations automatically generated by the Tensor Contraction Engine ${ }^{128,129}$ is an example of a successful co-design effort.

a. Closed-shell $\operatorname{CCSD}(T)$. The coupled-cluster method was introduced to chemistry by Ćížek ${ }^{130}$ (see also Ref. 131) and is a post-Hartree-Fock electron correlation method. Development of the canonical coupled-cluster code in NWChem commenced in 1995 under a collaboration with CRAY Inc. to develop a massively parallel coupled-cluster program designed to run on a CRAY T3E. Full details of the implementation are given in the work of Kobayashi and Rendell. ${ }^{13}$

The coupled-cluster wavefunction is written as an exponential of excitation operators acting on the reference determinant,

$$
\left|\Psi_{\mathrm{CC}}\right\rangle=e^{T}|\Phi\rangle
$$

where $T=T_{1}+T_{2}+\cdots$ is a cluster operator represented as a sum of its many-body components, i.e., singles $T_{1}$, doubles $T_{2}$, etc., and $|\Phi\rangle$ is the so-called reference function (usually chosen as a HartreeFock determinant). In practical applications, the above sum is truncated at some excitation rank. For example, the CCSD method ${ }^{30}$ is defined by including singles and doubles, i.e., $T \simeq T_{1}+T_{2}$. Introducing the exponential Ansatz (4) into the Schrödinger equation, premultiplying both sides by $e^{-T}$, using the Hausdorff formula, and projecting onto the subspace of excitation functions give a set of coupled non-linear equations that are solved iteratively to yield the coupled-cluster energy and amplitudes. For example, for the CCSD formulation, one obtains

$$
\begin{gathered}
\left\langle\Phi\left|\left(H_{N} e^{T_{1}+T_{2}}\right)_{C}\right| \Phi\right\rangle=\Delta E_{\mathrm{CCSD}}, \\
\left\langle\Phi_{i}^{a}\left|\left(H_{N} e^{T_{1}+T_{2}}\right)_{C}\right| \Phi\right\rangle=0, \\
\left\langle\Phi_{i j}^{a b}\left|\left(H_{N} e^{T_{1}+T_{2}}\right)_{C}\right| \Phi\right\rangle=0,
\end{gathered}
$$

where $H_{N}$ is the electronic Hamiltonian in the normal product form $\left(H_{N}=H-\langle\Phi|H| \Phi\rangle\right)$, subscript $C$ represents a connected part of a given operator expression, and $\Delta E_{\mathrm{CCSD}}$ is the CCSD correlation energy. The closed-shell CCSD implementation employs the optimized form of the CC equations discussed by Scuseria et al., ${ }^{133}$ as was programmed in the TITAN program. ${ }^{134}$ The nature of the CRAY T3E hardware required significant rewriting of earlier coupled-cluster algorithms to take into account the limited memory available per core $(8 \mathrm{MW})$ and the prohibitive penalty of $\mathrm{I} / \mathrm{O}$ operations. Of the various four indexed quantities, those with four occupied indices were replicated in the local memory (i.e., the memory associated with a single core) and those with one or two virtual indices were distributed across the global memory of the machine (i.e., the sum of the memory of all the processors) and accessed in computational batches. The terms involving integrals with three and four virtual orbital indices still proved too costly for the available memory and to circumvent this problem, these terms were evaluated in a "direct" fashion. This structure distinguishes NWChem from most other coupled-cluster programs. Thus, to make effective use of the available memory, as much as possible should be allocated, by using global arrays, with the bare minimum for the arrays replicated in local memory.

The canonical CCSD implementation in NWChem also contains the perturbative triples correction, denoted as $(\mathrm{T})$, of Raghavachari et al. ${ }^{31}$ This correction is an estimate from MøllerPlesset perturbation theory ${ }^{123}$ and evaluates the triples contribution to MP4 using the optimized cluster amplitudes at the end of a CCSD calculation. The $\operatorname{CCSD}(\mathrm{T})$ method is commonly referred to as the gold standard for $a b$ initio electronic structure theory calculations. Its computational cost scales as $n^{7}$, making it considerably more expensive than a CCSD calculation. However, the triples are noniterative and only require two-electron integrals with at most three virtual orbital indices, hence avoiding the previous memory and I/O issues, and so, the correction was easily adapted from the "aijkbc algorithm" of an earlier work by Rendell et al. ${ }^{13}$

In recent years, a great deal of effort was invested to enhance the performance of the iterative and non-iterative parts of the CCSD $(\mathrm{T})$ workflow. Performance tuning of the iterative part resulted in scaling the code up to 223200 processors of the ORNL Jaguar computer. ${ }^{26,136}$ Significant speed-ups for the CCSD iterative part were achieved by introducing efficient optimization techniques to alleviate the communication bottlenecks caused by a copious amount of communication requests introduced by a large class of lowdimensionality tensor contractions. This optimization provided a significant twofold to fivefold performance increase in the CCSD iteration time depending on the problem size and available memory and improved the CCSD scaling to 20000 nodes of the NCSA Blue Waters supercomputer. ${ }^{13}$ 
b. Tensor contraction engine and high-accuracy formulations. NWChem implements a large number of high-rank electroncorrelation methods for the ground, excited, and electrondetached/attached states as well as for molecular properties. The underlying Ansätze span configuration interaction (CI), coupledcluster (CC), many-body perturbation theories (MBPT), and various combinations thereof. A distinguishing feature of these implementations is their uniquely forward-looking development strategy. These parallel-executable codes, as well as their formulations and algorithms, were computer-generated by the symbolic algebra program $^{138}$ called the Tensor Contraction Engine (TCE). ${ }^{128}$ TCE was one of the first attempts to provide a scalable tensor library for parallel implementations of many-body methods, which extends the ideas of automatic CC code generation introduced by Janssen and Schaefer, ${ }^{139} \mathrm{Li}$ and Paldus, ${ }^{140}$ and Nooijen and Lotrich. ${ }^{141,142}$

The merits of such a symbolic system are many: (1) It expedites otherwise time-consuming and error-prone derivation and programming processes, (2) it facilitates parallelization and other laborious optimizations of the synthesized programs, (3) it enhances the portability, maintainability, extensibility and thus the lifespan of the whole program module, and (4) it enables new or higher-ranked methods to be implemented and tested rapidly, which are practically impossible to write manually. TCE is, therefore, one of the earliest examples ${ }^{139}$ of an expert system that lifts the burden of derivation/programming labor so that computational chemists can focus on imagining new Ansatz-a development paradigm embraced quickly by other chemistry software developers. $^{143-145}$

The working equations of an $a b$ initio electron-correlation method are written with sums-of-products of matrices, whose elements are integrals of operators in the Slater determinants. For many methods, the matrices have the general form ${ }^{146}$

$$
\left\langle\Phi_{i}\left|\hat{L}_{j}^{\dagger} \hat{H} \exp \left(\hat{T}_{k}\right) \hat{R}_{l}\right| \Phi_{m}\right\rangle_{\mathrm{C} / \mathrm{L}}
$$

where $\Phi_{i}$ is the whole set of the $i$-electron excited (or electrondetached/attached) Slater determinants, $\hat{H}$ is the Hamiltonian operator, $\hat{T}_{k}$ is a $k$-electron excitation operator, $\hat{R}_{l}$ is an $l$-electron excitation (or electron detachment/attachment) operator, and $\hat{L}_{j}^{\dagger}$ is a j-electron de-excitation (or electron detachment/attachment) operator. Subscript " $\mathrm{C} / \mathrm{L}$ " means that the operators can be required to be connected and/or linked diagrammatically. For example, the socalled $T_{2}$-amplitude equation of coupled-cluster singles and doubles (CCSD) is written as

$$
0=\left\langle\Phi_{2}\left|\hat{H} \exp \left(\hat{T}_{1}+\hat{T}_{2}\right)\right| \Phi_{0}\right\rangle_{\mathrm{C}}
$$

With the Ansatz of a method given in terms of Eq. (8), TCE (1) evaluates these operator-determinant expressions into sums-ofproducts of matrices (molecular integrals and excitation amplitudes) using normal-ordered second quantization and Wick's theorem, (2) transforms the latter into a computational sequence (algorithm), which consists in an ordered series of binary matrix multiplications and additions, and (3) generates parallel-execution programs implementing these matrix multiplications and additions, which can be directly copied into appropriate directories of the NWChem source code and which are called by a short, high-level driver subroutine humanly written (see Fig. 1).

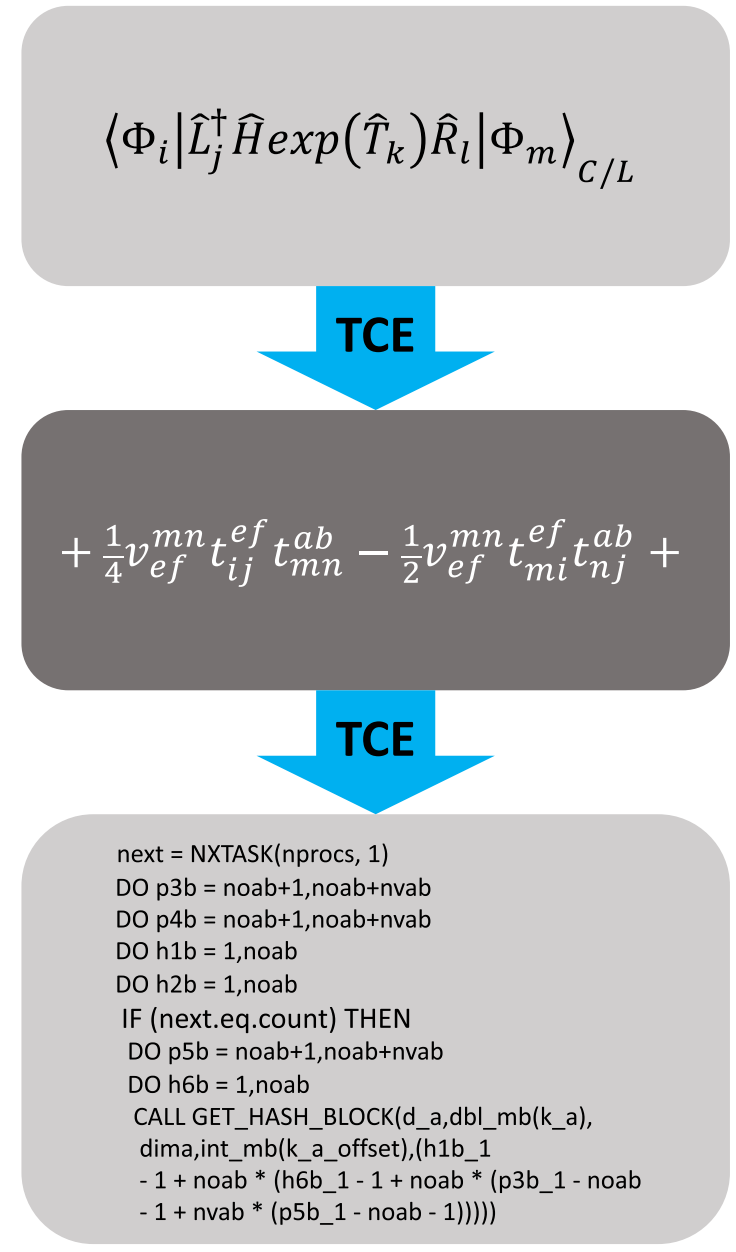

FIG. 1. A schematic representation of the TCE workflow (see the text for details).

In step (2), TCE finds the (near-)minimum cost path of evaluating sums-of-products of matrices by solving the matrix-chain problem (defining the so-called "intermediates") and by performing common subexpression elimination and intermediate reuse. In step (3), the computer-generated codes perform dynamically load-balanced parallel matrix multiplications and additions, taking advantage of spin, spatial, and index-permutation symmetries. The parallelism, symmetry usage, and memory/disk space management are all achieved by virtue of TCE's data structure: every matrix (molecular integrals, excitation amplitudes, intermediates, etc.) is split into spin- and spatial-symmetry-adapted tiles, whose sizes are determined at runtime so that the several largest tiles can fit in the core memory. Only symmetrically unique, non-zero tiles are stored gapless (with their storage addresses recorded in hash tables, which are also auto-generated by TCE) and used in parallel tile-wise multiplications and additions, which are dynamically distributed to idle processors on a first-come, first-served basis. NWChem's parallel middleware, especially global arrays, was essential for making the computer-generated parallel codes viable. 
TCE is a part of the NWChem source-code distribution, and a user is encouraged to implement their own Ansätze into highquality parallel codes. Therefore, TCE has paved the way for quick development of various implementations of coupled-cluster methods that would take disproportionately longer time if hand-coded. Additionally, TCE provided a new testing ground for several novel parallel algorithms for accurate many-body methods and has been used to generate a number of canonical implementations of single reference CC methods for ground-and excited-state calculations for arbitrary reference functions including RHF, ROHF, UHF, and multi-reference cases. In the following, we listed basic components of the TCE infrastructure in NWChem:

- various perturbative methods ranging from second [MBPT(2)/MP2] to fourth-order [MBPT(4)/MP4] of MöllerPlesset perturbation theory;

- single reference iterative (CCD ${ }^{130} \mathrm{CCSD}^{30} \mathrm{CCSDT}^{147-149}$ and $\operatorname{CCSDTQ}^{150,151}$ ) and non-iterative $\left[\operatorname{CCSD}(\mathrm{T}),{ }^{31} \mathrm{CR}\right.$ $\operatorname{CCSD}(\mathrm{T}),{ }^{152} \mathrm{LR}-\mathrm{CCSD}(\mathrm{T}),{ }^{153} \operatorname{CCSD}(2),{ }^{154-156} \operatorname{CCSD}(2){ }_{T},{ }^{156}$ and $\left.\operatorname{CCSDT}(2)_{Q}\right]^{156} \mathrm{CC}$ approximations for ground-state calculations;

- single reference iterative (EOMCCSD, ${ }^{157,158}$ EOMCCSDT, ${ }^{159,160}$ and EOMCCSDTQ ${ }^{146,161}$ ) and noniterative [CR-EOMCCSD $(\mathrm{T})^{162}$ ] equation-of-motion CC (EOMCC) approximations ${ }^{163}$ for excited-state calculations;

- ionization-potential and electron-affinity EOMCC (IP/EAEOMCC) methods; ${ }^{164-170}$

- linear-response CC (LR-CC) methods for calculating static and frequency-dependent polarizabilities and static hyperpolarizabilities at the CCSD and CCSDT levels of approximation; ${ }^{171}$ and

- state-specific multi-reference CC (MR-CC) methods for quasi-degenerate systems. ${ }^{172-178}$

The TCE infrastructure has also been used in exploring new parallel algorithms and algorithms for emerging computer architectures. The most important examples include

- parallel algorithms for excited-state CR-EOMCCSD(T) calculations with demonstrated scalability across 210000 cores of the Jaguar Cray XT5 system at the Oak Ridge Leadership Computing Facility (OLCF),

- new CC algorithms for GPU and Intel MIC architectures (single-reference CC and MR-CC theories), 32,34,35,179,180

- new algorithms for multi-reference CC methods utilizing processor groups and multiple levels of parallelism (the socalled reference-level of parallelism of Refs. 181 and 182) with demonstrated scalability across 80000 cores of the Jaguar Cray XT5 system, ${ }^{182}$ and

- new execution models for the iterative CCSD and EOMCCSD models.

With TCE, one can perform CC calculations for closed- and open-shell systems characterized by 1000-1300 orbitals. Some of the most illustrative examples of TCE calculations are (1) static and frequency-dependent polarizabilities for the $\mathrm{C}_{60}$ molecule, ${ }^{18}$ excited state simulations for $\pi$-conjugated chromophores, ${ }^{184}$ and IP-EOMCCSD calculations for ferrocene with explicit inclusion of solvent molecules. One cutting edge application of TCE CC was the early application of EOMCC methodologies in excited-state studies of functionalized forms of porphyrin. ${ }^{28}$ Additionally, TCE has also served as a development platform for early implementations of the coupled-cluster Green's function formalism. ${ }^{185-188}$ The TCE development has since been followed by several other efforts toward enabling scalable tensor libraries. This includes Super Instruction Assembly Language (SIAL), ${ }^{144,189}$ Cyclop Tensor Framework (CTF) ${ }^{190}$ TiledArray framework, ${ }^{191}$ and Libtensor, ${ }^{192}$ which have been used to develop scalable implementations of CC methods.

\section{Relativistic methods}

Methods that include treatment of relativistic effects are based on the Dirac equation, ${ }^{193}$ which has a four-component wavefunction. The solutions to the Dirac equation describe both positrons (the "negative energy" states) and electrons (the "positive energy" states), as well as both spin orientations and hence the four components. The wavefunction may be broken down into two-component functions traditionally known as the large and small components; these may further be broken down into the spin components. ${ }^{194-197}$

The implementation of approximate all-electron relativistic methods in quantum chemical codes requires the removal of the negative energy states and the factoring out of the spin-free terms. Both of these may be achieved using a transformation of the Dirac Hamiltonian known in general as a Foldy-Wouthuysen (FW) transformation. Unfortunately, this transformation cannot be represented in a closed form for a general potential and must be approximated. One popular approach is the Douglas and Kroll ${ }^{198}$ method developed by Hess. ${ }^{199,200}$ This approach decouples the positive and negative energy parts to second-order in the external potential (also to the fourth-order in the fine structure constant, $\alpha)$. Other approaches include the zeroth order regular approximation (ZORA), ${ }^{201-204}$ modification of the Dirac equation by Dyall, ${ }^{205}$ which involves an exact FW transformation on the atomic basis set level, ${ }^{206,207}$ and the exact 2-component (X2C) formulation, which is a catch-all for a variety of methods that arrive at an exactly decoupled two-component Hamiltonian using matrix algebra. ${ }^{197,208-211}$ NWChem contains released implementations of the DKH, ZORA, and Dyall approaches, while the $\mathrm{X} 2 \mathrm{C}$ method is available in a development version. ${ }^{209,211}$

Since these approximations only modify the integrals, they can, in principle, be used at all levels of theory. At present, the DouglasKroll, ZORA and X2C implementations can be used at all levels of theory, whereas Dyall's approach is currently available at the Hartree-Fock level.

\section{Douglas-Kroll approximation}

NWChem contains three second-order Douglas-Kroll approximations termed FPP, DKH, and DKHFULL. The FPP is the approximation based on free-particle projection operators, ${ }^{199}$ whereas the DKH and DKFULL approximations are based on external-field projection operators. ${ }^{200}$ The latter two are considerably better approximations than the former. DKH is the Douglas-Kroll-Hess approach and is the approach that is generally implemented in quantum chemistry codes. DKFULL includes certain cross-product integral terms ignored in the DKH approach (see Ref. 212). The third-order Douglas-Kroll approximation (DK3) implements the method by Nakajima and Hirao. 213,214 


\section{Zeroth order regular approximation (ZORA)}

The spin-free and spin-orbit versions of the one-electron zeroth order regular approximation (ZORA) have been implemented. Since the ZORA correction depends on the potential, it is not gauge invariant. This is addressed by using the atomic approximation of van Lenthe and co-workers. ${ }^{215,216}$ Within this approximation, the ZORA corrections are calculated using the superposition of densities of the atoms in the system. As a result, only intra-atomic contributions are involved, and no gradient or second derivatives of these corrections are needed to be calculated. In addition, the corrections need only to be calculated once at the start of the calculation and are stored. The ZORA approach is implemented in two ways in NWChem, one where the ZORA potential components are directly computed on an all-electron grid ${ }^{204}$ and a second approach where the ZORA potential is computed using the model potential approach due to van Wüllen and Michauk. ${ }^{217,218}$

\section{Dyall's modified Dirac Hamiltonian approximation}

The approximate methods described in this section are all based on Dyall's modified Dirac Hamiltonian. This Hamiltonian is entirely equivalent to the original Dirac Hamiltonian, and its solutions have the same properties. The modification is achieved by a transformation on the small component. This gives the modified small component the same symmetry as the large component. The advantage of the modification is that the operators now resemble those of the Breit-Pauli Hamiltonian and can be classified in a similar fashion into spin-free, spin-orbit, and spin-spin terms. It is the spin-free terms that have been implemented in NWChem, with a number of further approximations. Negative energy states are removed by a normalized elimination of the small component (NESC), which is equivalent to an exact Foldy-Wouthuysen (EFW) transformation. Both one-electron and two-electron versions of NESC (NESC1E and NESC2E, respectively) are available, and both have analytic gradients. $^{205-207}$

\section{E. Molecular properties}

A broad array of simple and response-based molecular properties can be calculated using the HF and DFT wavefunctions in NWChem. These include natural bond analysis, dipole, quadrupole, octupole moments, Mulliken population analysis and bond order analysis, Löwdin population analysis, electronic couplings for electron transfer, ${ }^{84,85}$ Raman spectroscopy, ${ }^{219,220}$ electrostatic potential (diamagnetic shielding) at nuclei, electric field and field gradient at nuclei, electric field gradients with relativistic effects, ${ }^{221}$ electron and spin density at nuclei, GIAO-based nuclear magnetic resonance (NMR) properties such as shielding, hyperfine coupling (FermiContact and spin-dipole expectation values), indirect spin-spin coupling, ${ }^{222-224}$ G-shift, ${ }^{225}$ EPR, paramagnetic NMR parameters, ${ }^{226,227}$ and optical activity. ${ }^{102,103,228,229}$ Note that only linear-response is supported for single frequency, electric field, and mixed electricmagnetic field perturbations. Ground state and dynamic dipole polarizabilities for molecules can be calculated at the CCSD, CCSDT, and CCSDTQ levels using the linear-response formalism. ${ }^{230}$ For additional information, we refer to the reader to the online manual.

\section{F. Periodic plane wave density functional theory}

The NWChem plane wave density functional theory (NWPW) module contains two programs:

- PSPW-a pseudopotential and projector augmented (PAW) plane wave $\Gamma$-point code for calculating molecules, liquids, crystals, and surfaces and

- BAND-a pseudopotential plane wave band structure code for calculating crystals and surfaces with small band gaps (e.g., semi-conductors and metals).

These programs use a common infrastructure for carrying out operations related to plane wave basis sets that are parallelized with the MPI and OpenMP libraries ${ }^{29,33,34,231-235}$ The NWPW module can be used to carry out many different kinds of simulations. In addition to the standard simulations implemented in other modules, e.g., energy, optimize, and frequency, there are additional capabilities specific to PSPW and BAND that can be used to carry out NVE and $\mathrm{NVT}^{236}$ Car-Parrinello ${ }^{19}$ and Born-Oppenheimer molecular dynamics simulations, hybrid $a b$ initio molecular dynamics molecular and molecular mechanics (AIMD-MM) simulations, ${ }^{234,237}$ Gaussian/Fermi/Marzari-Vanderbilt smearing, Potential-of-MeanForce $\quad(\mathrm{PMF})^{238} /$ Metadynamics $^{239,240} /$ Temperature-AcceleratedMolecular-Dynamics (TAMD) ${ }^{241,242} /$ Weighted-Histogram-AnalysisMethod (WHAM) ${ }^{243}$ free energy simulations, AIMD-EXAFS simulations using open source versions of the FEFF software ${ }^{244-246}$ that have been parallelized, electron transfer calculations, ${ }^{247}$ unit cell optimization, optimizations with space-group symmetry, MonteCarlo NVT and NPT simulations, phonon calculations, simulations with spin-orbit corrections, Wannier ${ }^{248}$ and rank reducing density matrix $^{249}$ localization calculations, Mulliken ${ }^{250}$ and Blöchl ${ }^{251}$ charge analysis, Gaussian cube file generation, periodic dipole and infrared (AIMD-IR) simulations, band structure plots, and density of states. Calculations can also be run using a newly developed i- $\mathrm{PI}^{252}$ interface, and more direct interfaces to ASE, ${ }^{253}$ nanoHUB, ${ }^{254}$ and EMSL Arrows $^{255}$ simulation tools are currently being implemented.

A variety of exchange-correlation functionals have been implemented in both codes, including the local density approximation (LDA) functionals, generalized gradient approximation (GGA) functionals, full Hartree-Fock and screened exchange, hybrid DFT functionals, self-interaction correction (SIC) functionals, ${ }^{256}$ localized exchange method, DFT + U method, and Grimme dispersion corrections, ${ }^{77,78}$ as well as recently implemented vdW dispersion functionals, ${ }^{257}$ and meta-generalized gradient approximation (metaGGA) functionals. The program contains several codes for generating pseudopotentials, including Hamann ${ }^{20}$ and TroulierMartins, ${ }^{21}$ and $\mathrm{PAW}^{23}$ potentials. These codes have the option for generating potentials with multiple projectors and semi-core corrections. It also contains codes for reading in $\mathrm{HGH},{ }^{258} \mathrm{GTH},{ }^{259}$ and norm-conserving pseudopotentials in the CPI and TETER formats. Codes for reading Optimized Norm-Conserving Vanderbilt (ONCV) pseudopotentials ${ }^{260,261}$ and USPP PAW potentials will become available in future releases of NWChem.

The pseudopotential plane wave DFT methods implemented in NWChem are a fast and efficient way to calculate molecular and solid-state properties using DFT. ${ }^{16,17,19,29,235,262-270}$ In these approaches, the fast varying parts of the valence wavefunctions inside the atomic core regions and the atomic core wavefunc- 


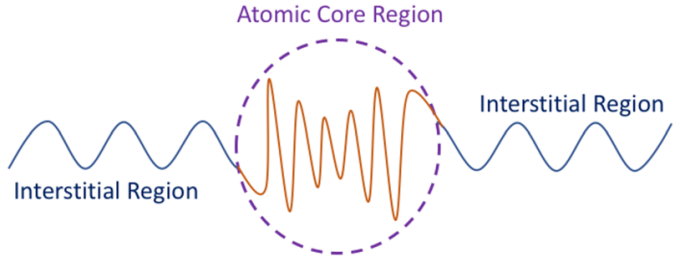

FIG. 2. Illustration of the atomic core and interstitial regions in a valence wavefunction. Bonding takes place in the interstitial region and the atomic core regions change very little from molecule to molecule. Figure from Bylaska et al. ${ }^{23}$

tions are removed and replaced by pseudopotentials. ${ }^{20-22,271-274}$ Pseudopotentials are chosen such that the resulting pseudoatoms have the same scattering properties as the original atoms. The rationale for this approach is that the changes in the electronic structure associated with making and breaking bonds only occur in the interstitial region outside the atomic core regions (see Fig. 2). Therefore, removing the core regions should not affect the bonding of the system. For this approach to be useful, it is necessary for the pseudopotentials to be smooth in order for plane wave basis sets to be used. As the atomic potential becomes stronger, the core region becomes smaller and the pseudopotential grows steep. As a result, the pseudopotential can become very stiff, requiring large plane wave basis sets (aka cutoff energies), for the first-row transition metals atoms, the lanthanide atoms, and toward the right-hand side of the periodic table (fluorine).

The projected augmented plane wave method (PAW) is another related approach that removes many of the problems of the somewhat ad hoc nature of the pseudopotentials approach. However, in the PAW approach, instead of discarding the rapidly varying parts of the electronic functions, these are projected onto a local basis set (e.g., a basis of atomic functions) and no part of the electron density is removed from the problem. Another key feature of PAW is that by maintaining a local description of the system, the norm-conservation condition (needed for proper scattering from the core) can be relaxed, which facilitates the use of smaller plane wave basis sets (aka cutoff energies) then for many standard pseudopotentials. Historically, the PAW method was implemented as a separate program in the NWPW module, rather than being fully integrated into the PSPW and BAND codes. This separation significantly hindered its development and use. As of NWChem version 6.8 (released in 2017), the PAW approach has been integrated into the PSPW code, and it is currently being integrated into the BAND code. It will become available in future releases of NWChem.

In recent years, with advances in High-Performance Computing (HPC) algorithms and computers, it is now possible to run AIMD simulations up to $\sim 1 \mathrm{~ns}$ for non-trivial system sizes. As a result, it is now possible to effectively use freeenergy methods with AIMD and AIMD/MM approaches. Free energy approaches are useful for simulating reactions where traditional quantum chemistry approaches can be difficult to use and often require the expertise of a very experienced quantum chemist, e.g., reactions that are complex with concerted or multi-step components and/or interact strongly with the solvent. Recent examples include solvent coordination and hydrolysis of actinides metals $^{197,278-281}$ (see Fig. 3), hydrolysis of explosives, ${ }^{234}$ and

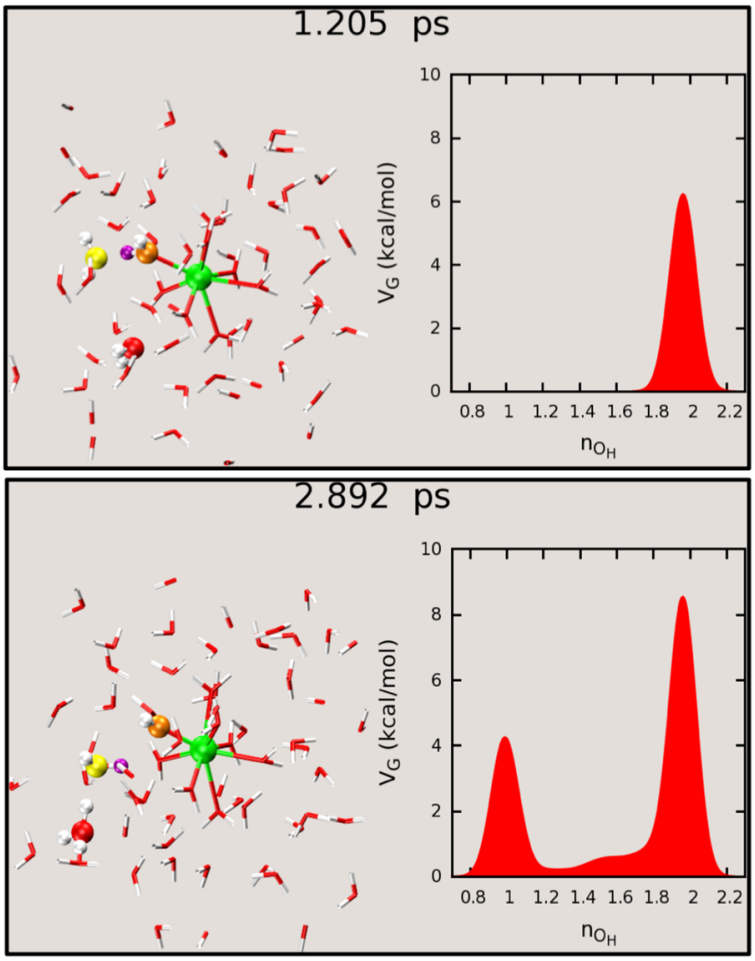

FIG. 3. Snapshots from a metadynamics simulation of the hydrolysis of the $U^{4+}$ aqua ion. ${ }^{278}$ During the simulation, a proton jumps from a first shell water molecule to a second shell water molecule and then subsequently to other water molecules via a Grotthuss mechanism.

ion association in $\mathrm{AlCl}_{3}{ }^{237}$ To help users learn how to use these new techniques, we developed a tutorial on carrying out finite temperature free energy calculations in NWChem.

The NWPW module continues to be actively developed. There are on-going developments for RPA and GW-RPA methods, an electron transfer MCSCF method, Raman and Mössbauer spectroscopy, and a hybrid method that integrates classical $\mathrm{DFT}^{283}$ into ab initio molecular dynamics (AIMD-CDFT). In addition to these developments, we are actively developing the next generation of plane wave codes as part of the NWChemEx project. These new codes, which are being completely written from scratch, will contain all the features currently existing in the NWPW module. Besides implementing fast algorithms to use an even larger number of cores and new algorithms to run efficiently on GPUs, it includes a more robust infrastructure to facilitate the implementation of an $O(N)$ DFT code based on the work of Osei-Kuffuor and Fattebert. ${ }^{28}$

\section{G. Optimization, transition state, and rate theory approaches}

A variety of drivers and interfaces are available in NWChem to perform geometry minimization and transition state optimizations. The default algorithms in NWChem for performing these optimizations are quasi-Newton methods with line searches. These methods 
are fairly robust, and they can be used to optimize molecules, clusters, and periodic unit cells and surfaces. They can also be used in conjunction with both point group and space group symmetries, excited state TDDFT surfaces, and with a variety of external fields, such as external point charges, COnductor-like Screening MOdel (COSMO) ${ }^{285}$ or Solvation Model based on Density (SMD). ${ }^{286}$ The default methods also work seamlessly with electronic structure methods that do not have nuclear gradients implemented by automatically using finite difference gradients. NWChem also contains default methods for calculating harmonic vibrational frequencies and phonon spectra for periodic systems. These methods are able to make use of analytic Hessians if they are available; otherwise, a finite difference approach is used. A vibrational selfconsistent field $^{287}$ (VSCF) method is also available in NWChem, and it can be used to calculate anharmonic contributions to specified vibrational modes. There is also an interface called DIRDYVTST ${ }^{288}$ that uses NWChem to compute energies, gradients, and Hessians for direct dynamics calculations with POLYRATE. ${ }^{289}$

A variety of external packages, such as $\operatorname{ASE}^{253,290}$ and Sella, ${ }^{291,292}$ can also be used for finding energy minima, saddle points on energy surfaces, and frequencies using either python scripting or a newly developed i- $\mathrm{PI}^{252}$ interface. Python programs may be directly embedded into the NWChem input and used to control the execution of NWChem. The python scripting language provides useful features, such as variables, conditional branches, and loops, and is also readily extended. Other example applications for which it could be used include scanning potential energy surfaces, computing properties in a variety of basis sets, optimizing the energy with respect to parameters in the basis set, computing polarizabilities with a finite field, simple molecular dynamics, and parallel in time molecular dynamics. ${ }^{2}$

NWChem also contains an implementation of the nudged elastic band (NEB) method of Jónsson and co-workers ${ }^{294-297}$ and the zero-temperature string method of Vanden Eijden et al. ${ }^{298}$ Both these methods can be used to find minimum energy paths. Currently, a quasi-Newton algorithm is used for the NEB optimization. A better approach for this kind of optimization is to use a nonlinear multi-grid algorithm, such as the Full Approximation Scheme (FAS). ${ }^{299}$ A new implementation of NEB based on FAS is available on Bitbucket, ${ }^{300}$ and an integrated version will be soon available in NWChem.

\section{H. Classical molecular dynamics}

The integration of a molecular dynamics (MD) module in NWChem enables the generation of time evolution trajectories based on Newton's equation of motion of molecular systems in which the required forces can originate from a classical force field, any implemented quantum mechanical method for which spatial derivatives have been implemented, or hybrid quantum mechan$\mathrm{ical} /$ molecular mechanical (QM/MM) approaches. The method is based on the ARGOS molecular dynamics software, originally designed for vector processors ${ }^{301}$ but later redesigned for massively parallel architectures. $25,302-304$

\section{System preparation}

The preparation of a molecular system is done by a separate prepare module that reads the molecular structure and assembles a topology from the databases with parameters for the selected force field. The topology file contains all static information for the system. In addition, this module generates the so-called restart file with all dynamic information. The prepare module has a wide range of capabilities that include the usual functions of placing counter-ions and solvation with any solvent defined in the database. The prepare module is also used to define Hamiltonian changes for free energy difference calculations and the definition of those parts of the molecular systems that will be treated quantum-mechanically in QM/MM simulations. Some of the more unique features include setting up a system for quantum mechanically derived proton hopping (QHOP) simulations ${ }^{305,306}$ and the setup of biological membranes from a single lipid-like molecule. This last capability has been successfully used for the first extensive simulation studies of complex asymmetric lipopolysaccharide membranes of Gram-negative microbes ${ }^{307-311}$ and their role in the capture of recalcitrant environmental heavy metal ions, ${ }^{312}$ microbial adhesion to geochemical surfaces, ${ }^{313-316}$ and the structure and dynamics of trans-membrane proteins including ion transporters (Fig. 4). ${ }^{317-319}$

\section{Force fields}

The force field implemented in NWChem consists of harmonic terms for bonded, angle and out of plane bending interactions, and trigonometric terms for torsions. Non-bonded van der Waals and electrostatic interactions are represented by Lennard-Jones and Coulombic terms, respectively. Non-bonded terms are evaluated using charge groups and subject to a user-specified cutoff radius. Electrostatic interaction corrections beyond the cutoff radius are estimated using the smooth particle mesh Ewald method. ${ }^{320}$ Parameter databases are provided for the $\mathrm{AMBER}^{321}$ and $\mathrm{CHARMM}^{322}$ force fields.

Even for purely classical MD simulations, the integration with the electronic structure methods provides a convenient way of determining electrostatic parameters for missing fragments in standard force field databases through the use of restrained electrostatic
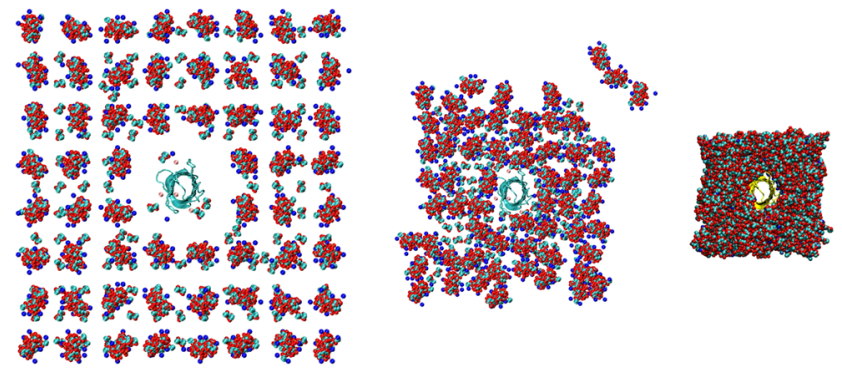

FIG. 4. The NWChem MD prepare utility facilitates the setup of trans-membrane proteins in complex asymmetric membrane environments in a semi-automated procedure. The top views of step 1 in which membrane lipopolysaccharide molecules with the necessary counter ions are placed on a rectangular grid around a trans-membrane protein in which each membrane lipid molecule is randomly rotated around the principal molecular axis (left panel), step 2 in which each cluster of a lipid molecule is translated toward the center of the transmembrane protein such that no steric clashes occur (center panel), and step 3 in which the system is equilibrated using strict restraint potentials to keep the lipid molecules aligned along the normal of the membrane and the lipid head groups in the plane of the membrane (right panel). After this procedure, the system would be solvated and equilibrated while slowly removing the positional restraint potentials. 
potential fitting ${ }^{323,324}$ to which a variety of additional constraints and restraints can be applied.

\section{Simulation capabilities}

Ensemble types available in NWChem are NVE, NVT, and NPT using the Berendsen thermostat and barostat. ${ }^{119}$ Newton's equations of motion are integrated using the standard leap-frog Verlet or velocity Verlet algorithms. A variety of fundamental properties are evaluated by default during any molecular dynamics simulation. Parallel execution time analysis is available to determine the parallel efficiency.

The MD module has extensive free energy simulation capabilities, ${ }^{325-330}$ which are implemented in the so-called multiconfiguration approach. For each incremental change in the Hamiltonian to move from the initial to the final state, sometimes referred to as a window, a full molecular simulation is carried out. This allows for a straightforward evaluation of statistical and systematic errors where needed, including a correlation analysis. ${ }^{331}$ Based on the ARGOS code, ${ }^{301}$ it has some unique features, such as the separation-shifted scaling technique to allow atoms to appear from or disappear to dummy atoms. ${ }^{332}$ One of the advantages of the integration of MD into the electronic structure methods framework in NWChem is the ability to carry out hybrid QM/MM simulations (discussed in Sec. VI). The preparation of molecular systems for the MD module allows for flexibly specifying parts of the molecular system to be treated by any of the implemented electronic structure methods capable of evaluating positional gradients.

A unique feature in the NWChem MD module is the optional specification of protonatable sites on both solute and solvent molecules. Pairs of such sites can dynamically change between protonated or unprotonated state, effectively exchanging a proton. Transitions are governed by a Monte Carlo type stochastic method to determine when transitions occur. This so-called QHOP approach was developed by the research group of Helms. ${ }^{306}$

\section{Analysis capabilities}

The NWChem MD capability includes two analysis modules. The original analysis module, analyze, analyzes trajectories in a way that reads individual structures one time step at a time and distributes the data in a domain decomposition fashion as in the molecular simulation that generated the data. The second data-intensive analysis module, diana, reads entire trajectories and distributes the data in the time domain. This is especially effective for analyses that require multiple passes through a trajectory but requires the availability of potentially large amounts of memory. ${ }^{333,334}$ An example of such analyses is the essential dynamics analysis, a principal component analysis (PCA) based calculation to determine the dominant motions in molecular trajectories.

\section{Parallel implementation strategy}

The most effective way of distributing a system with large numbers of particles is through the use of domain decomposition of the physical space. The implementation in NWChem, facilitated through the use of the Global Arrays (GA) toolkit, partitions the simulation space into rectangular cells that are assigned to different processes' ranks or threads. Each of these ranks carries out the calculation of intra-cell atomic energies and forces of the cells assigned. Inter-cell energies and forces are evaluated by one of the ranks that was assigned one or the other of the cell pairs.

Two load balancing methods have been implemented in NWChem, both based on measured computation time. In the first one, the assignment of inter-node cell pair calculations is redefined such that assignments move from the busiest node to the less busy node. This scheme requires minimal additional communication, and since only two nodes are involved in the redistribution of work, the communication is local, i.e., node to node. In the second scheme, the physical size of the most time-consuming cell is reduced, while all other cells are made slightly larger. This scheme requires communication and redistribution of atoms on all nodes. In practice, the first scheme is used until performance no longer improves, after which the second scheme is used once followed by returning to use the first scheme. This approach has been found to improve load balancing even in systems with a very asymmetric distribution of computational intensity.

\section{HYBRID METHODS}

We define hybrid methods as those coupling different levels of description to provide an efficient calculation of a chemical system, which otherwise may be outside the scope of conventional single-theory approaches. The physical motivation for such methods rests on the observation that, in the majority of complex chemical systems, the chemical transformation occurs in localized regions surrounded by an environment, which can be considered chemically inert to a reasonable approximation. Since hybrid methods require the combination of multiple theoretical methods in a single simulation, the diversity of simulation methodologies available in NWChem makes it a platform particularly apt for this purpose.

One common example involves chemical transformations in a bulk solution environment, forming the foundations of a wide variety of spectroscopic measurements (UV-vis, NMR, EPR, etc.). The reactive region, referred to as the "solute," involves electronic structure degrees of freedom and thus requires the quantummechanical (QM) based description, such as DFT or more complex wavefunction methods. In the conventional approach, such QM description would be necessarily extended to the entire system, making the problem a heroic computational task. In a hybrid approach, the treatment of a surrounding environment ("solvent") would be delegated to a much simpler description, such as the continuum model (CM). The latter is supported in NWChem via two models-COSMO ${ }^{285}$ (COnductor-like Screening MOdel) and $\mathrm{SMD}^{286}$ (Solvation Model based on Density). The resulting QM/CM approaches are particularly well suited for accurate and efficient calculation of solvation free energies, geometries in solution, and spectroscopy in solution. The SMD model employs the Poisson equation with a non-homogeneous dielectric constant for bulk electrostatic effects and solvent-accessible-surface tensions for cavitation, dispersion, and solvent-structure effects, including hydrogen bonding. For spectroscopy in solution, the Vertical Excitation (or Emission) Model (VEM) has also been implemented for calculating the vertical excitation (absorption) or vertical emission (fluorescence) energy in the solution according to a two-timescale model of solvent polarization. ${ }^{336}$ 
For systems where an explicit solvation environment treatment is needed (for example, heterogeneous systems such as a protein matrix), NWChem provides a solution in terms of the combined quantum mechanics/molecular mechanics (QM/MM) approach. ${ }^{337,338}$ Here, the environment is described at the classical molecular mechanics level. This offers more fidelity compared with a continuum solvent description, while still keeping the computational costs down. The total energy of the system in the QM/MM approach can be represented as a sum of the energies corresponding to $\mathrm{QM}$ and $\mathrm{MM}$ regions,

$$
E(\mathbf{r}, \mathbf{R} ; \psi)=E_{q m}(\mathbf{r}, \mathbf{R} ; \psi)+E_{m m}(\mathbf{r}, \mathbf{R}),
$$

where $\psi$ denotes electronic degrees of freedom and $\mathbf{r}$, and $\mathbf{R}$ refer to nuclear coordinates of QM and MM regions, respectively. The QM energy term can be further decomposed into internal and external parts,

$$
E_{q m}[\mathbf{r}, \mathbf{R} ; \psi]=E_{q m}^{\mathrm{int}}[\mathbf{r} ; \psi]+E_{q m}^{e x t}[\mathbf{r}, \mathbf{R} ; \rho]
$$

where $\rho$ is the electron density.

As a generic module, the QM/MM implementation can utilize any of the Gaussian basis set based QM modules available in NWChem and supports nearly all the task functionalities. The calculation of QM energy remains the main computational expense in the $\mathrm{QM} / \mathrm{MM}$ approach. This issue is more pronounced compared with the continuum coupling case, because of the additional atomistic degrees of freedom associated with the MM description. The latter comes into play because any change in the MM degrees of freedom will, in general, trigger the recalculation of the QM energy $\left[E_{q m}(\mathbf{r}, \mathbf{R} ; \psi)\right]$. To alleviate these issues during the optimization, the QM/MM module offers the option of alternating relaxation of QM and MM regions. During the latter phase, the user may utilize an approximation where the QM degrees of freedom are kept frozen until the next cycle of QM region relaxation, offering significant computational savings. A similar technique can be utilized in the dynamical equilibration of the MM region and calculations of reaction pathways and free energies. In addition to the native MD module, the NWChem QM/MM module can also utilize the external AMBER MD code ${ }^{339}$ for running the classical part of the calculations. In this case, QM/MM simulations involve two separate NWChem and AMBER calculations with data exchange mediated through files written to the disk.

Additionally, the QM/MM capability in NWChem has resulted in the development and refinement of force-field parameters, which can, in turn, be used in classical molecular dynamics simulations. Over the last two decades, classical parameters obtained using NWChem have been employed to address the underlying mechanisms of a variety of novel complex biological systems and their interactions (e.g., lipopolysaccharide membranes, carbohydrate moieties, mineral surfaces, radionuclides, and organophosphorous compounds), ${ }^{307,308,311-313,340-344}$ which has led to a significant expansion of the database of AMBER- and Glycam-compatible force fields and the GROMOS force field for lipids, carbohydrates, and nucleic acids. ${ }^{345-351}$

For cases where a classical description of the environment is deemed insufficient, NWChem offers an option to perform an ONIOM type calculation. ${ }^{352}$ The latter differs from QM/MM in that the lower level of theory is not restricted to its region but also encompasses regions from all the higher levels of description. For example, in the case of the two-level description, the energy is written as

$$
E(\mathbf{R})=E^{L}(\mathbf{R})+\left(E^{H}\left(\mathbf{R}^{H}\right)-E^{L}\left(\mathbf{R}^{H}\right)\right),
$$

where subscripts $H$ and $L$ refer to high and low levels of theory, respectively. The high-level treatment is restricted to a smaller portion of the system $\left(\mathbf{R}^{H}\right)$, while the low level of theory goes over the entire space (R). The second term in the above equation takes care of overcounting. The NWChem ONIOM module implements twoand three-layer ONIOM models for use in energy, gradient, geometry optimization, and vibrational frequency calculations with any of the pure QM methods within NWChem.

A new development in hybrid method capabilities of NWChem involves classical density functional theory (cDFT). ${ }^{353-355}$ The latter represents a classical variant of electronic structure DFT, where the main variable is the classical density of the atoms. ${ }^{356,357}$ Conceptually, this type of description lies between continuum and classical force field models, providing orders of magnitude improvements over classical MD simulations. The approach is based on incorporating important structural features of the environment in the form of classical correlation functions. This allows for efficient and reliable calculations of thermodynamical quantities, providing an essential link between the electronic structure description at the atomistic level and phenomena observed at the macroscopic scale.

\section{PARALLEL PERFORMANCE}

The design and development of NWChem from the outset was driven by parallel scalability and performance to enable large scale calculations and achieve fast time-to-solution by using many CPUs where possible. The parallel tools outlined in Sec. IV provided the programming framework for this.

The advent of new architectures such as the GPU ${ }^{358}$ platforms has required the parallel coding strategy within NWChem to be revisited. At present, the coupled-cluster code within TCE can utilize both the CPU and GPU hardware at a massive scale. ${ }^{32,359}$ The emergence of many-core processors in the last ten years provided the opportunity for starting a collaborative effort with Intel corporation to optimize NWChem on this new class of computer architecture. As part of this collaboration, the TCE implementation of the $\operatorname{CCSD}(\mathrm{T})$ code was ported to the Intel Xeon Phi line of many-core processors ${ }^{35}$ using a parallelization strategy based on a hybrid GAOpenMP approach. The ab initio plane wave molecular dynamics code (Sec. V F) has also been optimized to take full advantage of these Intel many-core processors. ${ }^{33,231}$

In the rest of this section, we will discuss the parallel scalability and performance of the main capabilities in NWChem.

\section{A. Gaussian basis density functional theory}

In Fig. 5, we report the parallel performance of the Gaussian basis set DFT module in NWChem. This calculation involved performing a PBE0 energy calculation (four SCF iterations in direct mode) on the $\mathrm{C}_{240}$ molecule with the $6-31 \mathrm{G}^{*}$ basis set (3600 basis functions) without symmetry. These calculations were performed on the Cascade supercomputer located at PNNL. 


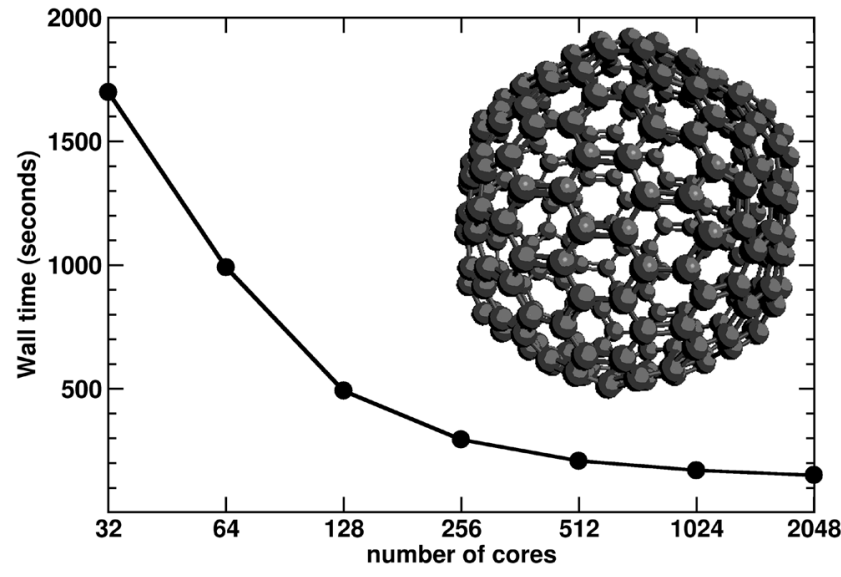

FIG. 5. $C_{240}$ DFT benchmark.

\section{B. Time-dependent density functional theory}

In Fig. 6, we report the parallel performance of the Gaussian basis set LR-TDDFT module in NWChem. This calculation involved computing 100 excitation energies, requiring 11 Davidson iterations, for the $\mathrm{Au}_{20}$ molecule surrounded by a matrix of $80 \mathrm{Ne}$ atoms $^{360}$ (1840 basis functions) with $D_{2}$ symmetry using the B3LYP functional. These calculations were performed on the Cascade supercomputer located at PNNL.

\section{Closed-shell CCSD(T)}

The parallel implementation of the $\operatorname{CCSD}(\mathrm{T})$ approach by Kobayashi and Rendell, ${ }^{132}$ employing the spin adaptation scheme based on the unitary group approach (UGA) ${ }^{133}$ within NWChem, was one of the first scalable implementations of the CC formalism capable of taking advantage of several hundred processors. This implementation was used in simulations involving tera- and petascale architectures where chemical accuracy is required to describe ground-state potential energy surfaces. One of the best illustrations

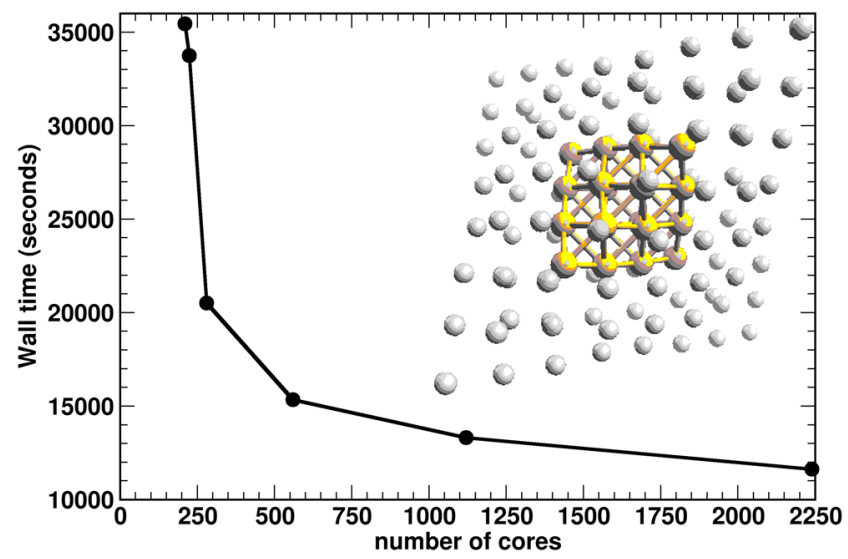

FIG. 6. LR-TDDFT benchmark for the $\mathrm{Au}_{20}$ molecule in a neon matrix. of the performance of the $\operatorname{CCSD}(\mathrm{T})$ implementation is provided by calculations for water clusters. ${ }^{26}$ In the largest calculation, $\left(\mathrm{H}_{2} \mathrm{O}\right)_{24}$, a sustained performance of $1.39 \mathrm{PetaFLOP} / \mathrm{s}$ (double precision) on 223200 processors of ORNL's Jaguar system was documented. This impressive performance was mostly attributed to the (T)-part characterized by $n_{o}^{3} n_{u}^{4}$ numerical overhead (where $n_{o}$ and $n_{u}$ refer to the total numbers of correlated occupied and virtual orbitals) and its relatively low communication footprint.

\section{Tensor contraction engine}

The TCE has enabled parallel CC/EOMCC/LR-CC calculations for closed- and open-shell systems characterized by 1000-1300 orbitals. Some of the most illustrative examples include calculations for static and frequency-dependent polarizabilities for polyacenes and $\mathrm{C}_{60}$ molecule, ${ }^{183,361}$ excited state simulations for $\pi$ conjugated chromophores, ${ }^{184}$ and IP-EOMCCSD calculations for carbon nanotubes. ${ }^{362}$ A good illustration of the scalability of the TCE module is provided by the application of GA-based TCE implementations of the iterative (CCSD/EOMCCSD) and non-iterative [CR$\operatorname{EOMCCSD}(\mathrm{T})]$ methods in studies of excited states of $\beta$-carotene ${ }^{363}$ and functionalized forms of porphyrin ${ }^{28}$ [see Figs. 7(a) and 7(b), respectively]. While non-iterative methods are much easier to scale across a large number of cores [Fig. 7(b)], scalability of the iterative CC methods is less easy to achieve. However, using early task-flow algorithms for TCE CCSD/EOMCCSD methods, ${ }^{28}$ it was possible to achieve satisfactory scalability in the range of 1000-8000 cores.

\section{E. Recent implementation of plane wave DFT AIMD for many-core architectures}

The very high degree of parallelism available on machines with many-core processors is forcing developers to carefully revisit the implementation of their programs in order to make use of this hardware efficiently. In this section, after a brief overview of the computational costs and parallel strategies for AIMD, we present our recent work ${ }^{33}$ on adding thread-level parallelism to the AIMD method implemented in NWChem. ${ }^{3,29,364}$

The main computational costs of an energy minimization or AIMD simulation are the evaluation of the electronic gradient $\delta E_{\text {total }} / \delta \psi_{i}^{*}=H \psi_{i}$ and algorithms used to maintain orthogonality. These costs are illustrated in Fig. 8. Due to their computational complexity, the electron gradient $H \psi_{i}$ and orthogonalization need to be calculated as efficiently as possible. The main parameters that determine the cost of a calculation are $N_{g}, N_{e}, N_{a}$, and $N_{\text {proj }}$, where $N_{g}$ is the size of the three-dimensional FFT grid, $N_{e}$ is the number of occupied orbitals, $N_{a}$ is the number of atoms, $N_{\text {proj }}$ is the number of projectors per atom, and $N_{\text {pack }}$ is the size of the reciprocal space.

The evaluation of the electron gradient (and orthogonality) contains three major computational pieces that need to be efficiently parallelized:

- applying $V_{H}$ and $V_{x c}$, involving the calculation of $2 N_{e} 3 \mathrm{D}$ FFTs;

- calculating the non-local pseudopotential, $V_{N L}$, dominated by the cost of the matrix multiplications $W=P^{T} Y$ and $Y_{2}=$ $P W$, where $P$ is an $N_{\text {pack }} \times\left(N_{\text {proj }} \cdot N_{a}\right)$ matrix, $Y$ and $Y_{2}$ are $N_{\text {pack }} \times N_{e}$ matrices, and $W$ is an $\left(N_{\text {proj }} N_{a}\right) \times N_{e}$ matrix; 

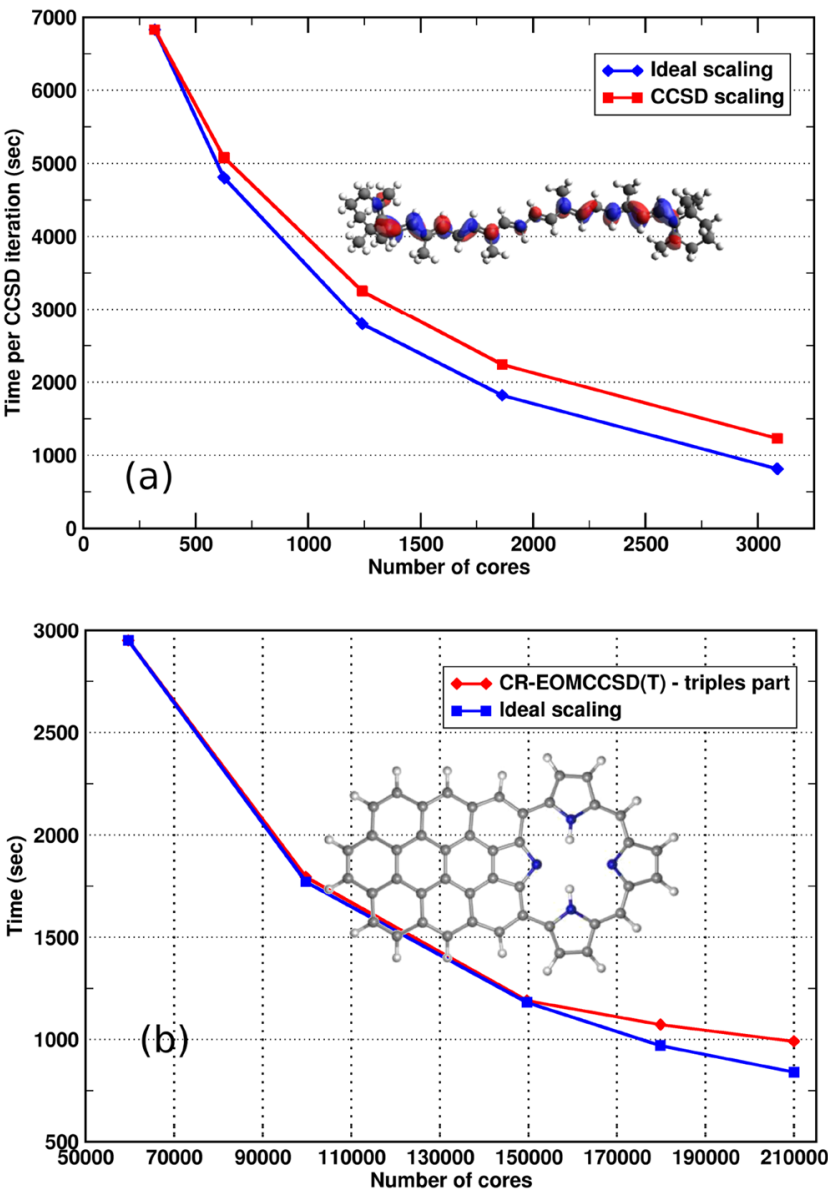

FIG. 7. Benchmark EOMCC scalability tests for (a) $\beta$-carotene and (b) free-base porphyrin (FBP) fused coronene. Timings for CR-EOMCCSD(T) approach for the coronene fused free-base porphyrin in the AVTZ basis set were determined from calculations on the ORNL's Jaguar Cray XT5 computer system.

$$
\begin{gathered}
-\frac{1}{2} \nabla^{2} \Psi+V_{e x t} \Psi+V_{H} \Psi+V_{x c} \Psi+V_{x, \text { exact }} \Psi=E \Psi \\
\langle\Psi \mid \Psi\rangle=\delta_{i, j}
\end{gathered}
$$

$$
\begin{aligned}
& \text { Approximate Number of Floating-Point Operations } \\
& -\frac{1}{2} \nabla^{2} \Psi: N_{\mathrm{e}} \mathrm{N}_{\mathrm{g}} \\
& V_{\text {ext }} \Psi:\left(\mathrm{N}_{\mathrm{A}} \mathrm{N}_{\mathrm{g}}+\mathrm{N}_{\mathrm{g}} \log \left(\mathrm{N}_{\mathrm{g}}\right)+\mathrm{N}_{\mathrm{e}} \mathrm{N}_{\mathrm{g}}\right)+\mathrm{N}_{\mathrm{A}} \mathrm{N}_{\text {prj }} \mathrm{N}_{\mathrm{g}} \\
& V_{H} \Psi: \mathrm{N}_{\mathrm{e}} \mathrm{N}_{\mathrm{g}} \log \left(\mathrm{N}_{\mathrm{g}}\right)+2 \mathrm{~N}_{\mathrm{e}} \mathrm{N}_{\mathrm{g}}+\mathrm{N}_{\mathrm{g}}+2 \mathrm{NgLog}\left(\mathrm{N}_{\mathrm{g}}\right) \\
& V_{x c} \Psi: \mathrm{N}_{\mathrm{e}} \mathrm{N}_{\mathrm{g}} \log \left(\mathrm{N}_{\mathrm{g}}\right)+\mathrm{N}_{\mathrm{e}} \mathrm{N}_{\mathrm{g}} \\
& V_{\text {x,exact }} \Psi: \mathrm{N}_{\mathrm{e}}\left(\mathrm{N}_{\mathrm{e}}+1\right) \mathrm{N}_{\mathrm{g}} \log \left(\mathrm{N}_{\mathrm{g}}\right) \\
& \langle\Psi \mid \Psi\rangle=\delta_{i, j}: \mathrm{N}_{\mathrm{e}}{ }^{2} \mathrm{~N}_{\mathrm{g}}+\mathrm{N}_{\mathrm{e}}{ }^{3} \\
& \mathrm{~N}_{\mathrm{A}} \text { - number of atoms } \\
& \mathrm{N}_{\text {pri }} \text { - average number of } \\
& \text { projectors per atom } \\
& \mathrm{N}_{\mathrm{e}} \text { - number of electrons } \\
& \mathrm{N}_{\mathrm{g}} \text {-number of grid points }
\end{aligned}
$$

FIG. 8. Operation count of $H_{\psi}$ in a plane wave DFT simulation. Figure from Ref. 231.
- enforcing orthogonality, where the most expensive matrix multiplications are $S=Y^{T} Y$ and $Y_{2}=Y S$, where $Y$ and $Y_{2}$ are $N_{\text {pack }} \times N_{e}$ matrices and $S$ is an $N_{e} \times N_{e}$ matrix. In this work, Lagrange multiplier kernels are used for maintaining orthogonality of Kohn-Sham orbitals. ${ }^{29,365-368}$

In Fig. 9, the timing results for a full AIMD simulation of 256 water molecules on $16,32,64,128,256$, and $1024 \mathrm{KNL}$ nodes are shown. The "Cori" system at NERSC was used to run this benchmark. This benchmark was taken from Car-Parrinello simulations of $256 \mathrm{H}_{2} \mathrm{O}$ with an FFT grid of $N_{g}=180^{3}\left(N_{e}=2056\right)$ using the plane wave DFT module (PSPW) in NWChem. In these timings, the number of threads per node was 66 . The size of this benchmark simulation is about four times larger than many mid-size AIMD simulations carried out in recent years, e.g., in recent work by Bylaska and co-workers. ${ }^{279,280,369-372}$ The overall timings show strong scaling up to $1024 \mathrm{KNL}$ nodes (69632 cores) and the timings of the major kernels, the pipelined 3D FFTs, non-local pseudopotential, and Lagrange multiplier kernels all displayed significant speedups.

\section{F. Classical molecular dynamics}

The molecular dynamics module in the current NWChem release is based on the distribution of cells over available ranks in the calculation. Simulations exhibit good scalability when cells only require communication with immediately neighboring cells. When the combination of the cell size and cutoff radius is such that interactions with atoms in cells beyond the immediate neighbors are required, performance is significantly affected. This limits the number of ranks that can effectively be used. For example, a system with 500000 atoms will only scale well up to 1000 ranks. In future implementations, the cell-cell pair-list will be distributed over the available ranks. While this leads to additional communication for ranks that do not "own" a cell, the implementation of a new communication scheme that avoids global communication has been demonstrated to improve scalability by at least an order of magnitude. ${ }^{304}$

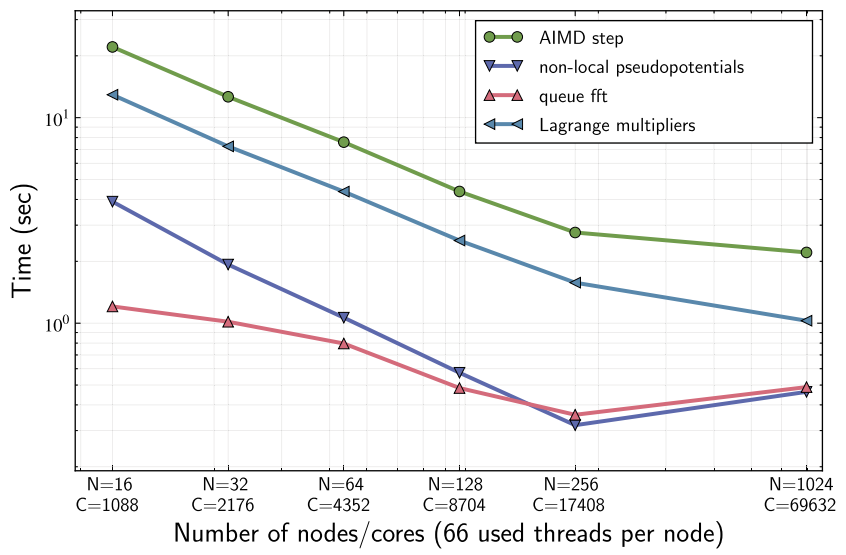

FIG. 9. Scalability of major components of an AIMD step on the Xeon Phi partition for a simulation of $256 \mathrm{H}_{2} \mathrm{O}$ molecules. Figure from Bylaska et al. 


\section{OUTREACH}

Given the various electronic structure methods available in NWChem, it does not come as a surprise that many of these functionalities have been integral to various projects focused on extensions of quantum chemical capabilities to exa-scale architectures and emerging quantum computing (see Fig. 10 for a pictorial representation of recent developments). In the following, we describe several examples of such a synergy.

\section{A. Interfacing with other software}

Over the years, many open-source and commercial developers have been using NWChem as a resource for their capability development and building add-on tools to increase the code's usability. Various open-source and commercial platforms provide user interface capabilities to setup and analyze the results of calculations that can be performed with NWChem. ${ }^{253,255,373-380}$ NWChem initially developed its own graphical user interface called the Extensible Computational Chemistry Environment, ${ }^{381}$ which is currently supported by a group of open-source developers. In addition, multiple codes use quantities from the NWChem simulation, such as wavefunctions as the input for the calculation of additional properties not directly available in the code. ${ }^{382-391}$ NWChem is able to export electrostatic potential and charge densities with the Gaussian cube format ${ }^{392}$ and can use the Molden format ${ }^{393}$ to write or read molecular orbitals. This allows codes ${ }^{394-398}$ to utilize NWChem's data to, for example, display charge densities and electrostatic potentials. NWChem can also generate AIM wavefunction files that have been used by a variety of codes to calculate various properties. ${ }^{76,399-401}$ Recently, NWChem has also been interfaced with the SEMIEMP code, ${ }^{402}$ which can be used to perform real-time electronic dynamics using the INDO/S Hamiltonian. ${ }^{403,404}$

\section{B. Common component architecture}

It is an attractive idea to encapsulate complex scientific applications as components with standardized interfaces. The components

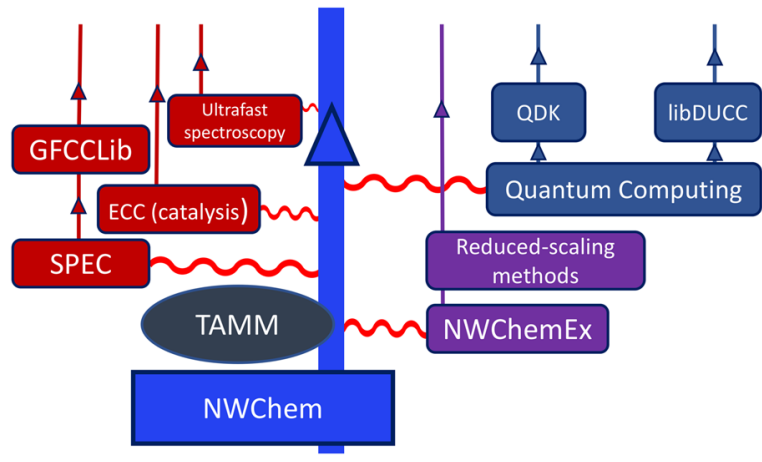

FIG. 10. A "connected diagram" describing ongoing efforts to extend computational chemistry models to exa-scale and quantum computing. In each case, NWChem provides a testing and development platform. A significant role in these projects is played by Tensor Algebra for the Many-body Methods (TAMM) library. The ECC acronym stands for the Exa-scale Catalytic Chemistry project supported by BES. ${ }^{411}$ The QDK-NWChem interface with the libDUCC library is used for downfolding electronic Hamiltonians. ${ }^{41}$ interact only through these well-defined interfaces and can be combined into full applications. The main motivation is to be able to reuse and swap components as needed and seamlessly create complex applications. There have been a few attempts to introduce this approach to the scientific software development community. The most notable DOE-led effort was the Common Component Architecture (CCA) Forum, ${ }^{405}$ which was launched in 1998 as a scientific community effort to create components designed specifically for the needs of high-performance scientific computing. A more recent development is the rise of Simulation Development Environment (SDE) framework, ${ }^{406}$ which has features that are related to the components of CCA.

NWChem developers have participated in CCA and SDE effort resulting in the creation of the NWChem component. As an example, the NWChem CCA component was used in the building applications for molecular geometry optimization from multiple quantum chemistry and numerical optimization packages, ${ }^{407}$ combination of multiple theoretical methods to improve multi-level parallelism, ${ }^{408}$ demonstration of multi-level parallelism, ${ }^{409}$ and standardization of integral interfaces in quantum chemistry. ${ }^{410}$ In the end, the CCA framework was too cumbersome to use for developers, requiring significant efforts to develop interfaces and making components to work together. It resulted in the retirement of CCA Forum in 2010, but the work done on standardization of interfaces is continuing to benefit the quantum chemistry community to this day.

\section{NWChemEx}

The NWChemEx project is a natural extension of NWChem to overcome the scalability challenges associated with the migration of the current code base to exa-scale platforms. NWChemEx is being developed to address two outstanding problems in advanced biofuels research: (i) development of a molecular understanding of proton controlled membrane transport processes and (ii) development of catalysts for the efficient conversion of biomass-derived intermediates into biofuels, hydrogen, and other bioproducts. Therefore, the main focus is on enabling scalable implementations of the groundstate canonical CC formalisms utilizing the Cholesky decomposed form of the two-electron integrals, ${ }^{413-418}$ as well as linear scaling CC formulations based on the domain-based local pair natural orbital CC formulations (DLPNO-CC) $)^{419-421}$ and embedding methods.

\section{Scalable predictive methods for excitations and correlated phenomena (SPEC)}

The main focus of the SPEC software project is to provide the users with a new generation of methodologies to simulate excited states and excited-state processes using existing peta- and emerging exa-scale architectures. These new capabilities will play an important role in supporting the experimental efforts at light source facilities, which require accurate and reliable modeling tools. The existing NWChem capabilities are being used to verify and validate SPEC implementations, including excitation energy, ionization potential, and electron affinity variants of the EOMCC theory as well as hierarchical Green's function formulations ranging from the lower order GW + Bethe-Salpeter equation $(\mathrm{GW}+\mathrm{BSE})^{422}$ to hierarchical coupled-cluster Green's function (GFCC) methods ${ }^{185-188,423}$ and multi-reference CC methods. 


\section{E. Quantum information sciences}

Quantum computing offers the promise of not only overcoming exponential computational barriers of conventional computing but also achieving the ultimate level of accuracy in studies of challenging processes involving multi-configurational states in catalysis, biochemistry, photochemistry, and materials science to name only a few areas where quantum information technologies can lead to the transformative changes in the way how quantum simulations are performed. NWChem, with its computational infrastructure to characterize second-quantized forms of electronic Hamiltonians in various basis sets (Gaussian and plane waves) and with wavefunction methodologies to provide an initial characterization of the groundand excited-state wavefunctions, can be used as a support platform for various types of quantum simulators. The recently developed QDK-NWChem interface ${ }^{424}$ (QDK designates Quantum Development Kit developed by Microsoft Research team) for quantum simulations and libraries for CC downfolded electronic Hamiltonians for quantum computing ${ }^{412}$ are good illustrations of the utilization of NWChem in supporting the quantum computing effort.

\section{CONCLUSIONS}

The NWChem project is an example of a successful co-design effort that harnesses the expertise and experience of researchers in several complementary areas, including quantum chemistry, applied mathematics, and high-performance computing. Over the last three decades, NWChem has evolved into a code that offers a unique combination of computational tools to tackle complex chemical processes at various spatial and time scales.

In addition to the development of new methodologies, NWChem is continuously upgraded with new algorithms to take advantage of emerging computer architectures and quantum information technologies. We believe that the community model of NWChem will continue to spur exciting new developments well into the future.

\section{SUPPLEMENTARY MATERIAL}

See the supplementary material for tutorial slides showing examples of NWChem input files.

\section{ACKNOWLEDGMENTS}

The authors acknowledge the important contributions to the NWChem project of the following deceased researchers: Ricky A. Kendall, Jarek Nieplocha, and Daniel W. Silverstein.

We would like to thank people who have helped the progress of the NWChem project by providing valuable feedback, scientific direction, suggestions, discussions, and encouragement. This (incomplete) list includes A. Andersen, J. Andzelm, R. Baer, A. Bick, V. Blum, D. Bowman, E. A. Carter, A. Chakraborty, G. Cisneros, D. Clerc, A. J. Cohen, L. R. Corrales, A. R. Felmy, A. Fortunelli, J. Fulton, D. A. Dixon, P. Z. El-Khoury, D. Elwood, G. Fanourgakis, D. Feller, B. C. Garrett, B. Ginovska, E. Glendening, V. A. Glezakou, M. F. Guest, M. Gutowski, M. Hackler, M. Hanwell, W. L. Hase, A. C. Hess, W. M. Holden, C. Huang, W. Huang, E. S. Ilton, E. P. Jahrman, J. Jakowski, J. E. Jaffe, J. Ju, S. M. Kathmann, R. Kawai, M. Khalil, X. Krokidis, L. Kronik, P. S. Krstić, R. Kutteh, X. Long, A. Migliore, E. Miliordos, M. Nooijen, J. Li, L. Lin, S. Liu, N. Maitra, M. Malagoli,
B. Moore II, P. Mori, S. Mukamel, C. J. Mundy, V. Meunier, V. Murugesan, D. Neuhauser, S. Niu, R. M. Olson, S. Pamidighantam, M. Pavanello, M. P. Prange, C. D. Pemmaraju, D. Prendergast, S. Raugei, J. J. Rehr, A. P. Rendell, R. Renslow, T. Risthaus, K. M. Rosso, R. Rousseau, J. R. Rustad, G. Sandrone, G. K. Schenter, G. T. Seidler, H. Sekino, P. Sherwood, C. Skylaris, H. Song, L. Subramanian, B. G. Sumpter, P. Sushko, S. Tretiak, R. M. Van Ginhoven, B. E. Van Kuiken, J. H. van Lenthe, B. Veeraraghavan, E. Vorpagel, X. B. Wang, J. Warneke, Y. A. Wang, J. C. Wells, S. S. Xantheas, W. Yang, J. Zador, and Y. Zhang.

The core development team acknowledges support from the following projects at the Pacific Northwest National Laboratory. Pacific Northwest National Laboratory is operated by Battelle Memorial Institute for the U.S. Department of Energy under Contract No. DE-AC05-76RL01830: (i) Environmental and Molecular Sciences Laboratory (EMSL), the Construction Project, and Operations, the Office of Biological and Environmental Research, (ii) the Office of Basic Energy Sciences, Mathematical, Information, and Computational Sciences, Division of Chemical Sciences, Geosciences, and Biosciences (CPIMS, AMOS, Geosciences, Heavy Element Chemistry, BES Initiatives: CCS-SPEC, CCS-ECC, BES-QIS, BES-Ultrafast), (iii) the Office of Advanced Scientific Computing Research through the Scientific Discovery through Advanced Computing (SciDAC), Exascale Computing Project (ECP): NWChemEx. Additional funding was provided by the Office of Naval Research, the U.S. DOE High Performance Computing and Communications Initiative, and industrial collaborations (Cray, Intel, Samsung).

The work related to the development of QDK-NWChem interface was supported by the "Embedding Quantum Computing into Many-body Frameworks for Strongly Correlated Molecular and Materials Systems" project, which is funded by the U.S. Department of Energy (DOE), Office of Science, Office of Basic Energy Sciences, the Division of Chemical Sciences, Geosciences, and Biosciences, and Quantum Algorithms, Software, and Architectures (QUASAR) Initiative at Pacific Northwest National Laboratory (PNNL).

S. A. Fischer acknowledges support from the U.S. Office of Naval Research through the U.S. Naval Research Laboratory.

A. J. Logsdail acknowledges support from the UK EPSRC under the "Scalable Quantum Chemistry with Flexible Embedding" (Grant Nos. EP/I030662/1 and EP/K038419/1).

A. Otero-de-la-Roza acknowledges support from the Spanish government for a Ramón y Cajal fellowship (No. RyC-2016-20301) and for financial support (Project Nos. PGC2018-097520-A-100 and RED2018-102612-T).

J. Autschbach acknowledges support from the U.S. Department of Energy, Office of Basic Energy Sciences, Heavy Element Chemistry program (Grant No. DE-SC0001136, relativistic methods \& magnetic resonance parameters) and the National Science Foundation (Grant No. CHE-1855470, dynamic response methods).

D. Mejia Rodriguez acknowledges support from the U.S. Department of Energy (Grant No. DE-SC0002139).

D. G. Truhlar acknowledges support from the NSF under Grant No. CHE-1746186.

E. D. Hermes was supported by the U.S. Department of Energy, Office of Science, Basic Energy Sciences, Chemical Sciences, Geosciences and Biosciences Division, as part of the Computational Chemistry Sciences Program (Award No. 0000232253). Sandia National Laboratories is a multimission laboratory managed 
and operated by National Technology and Engineering Solutions of Sandia, LLC, a wholly owned subsidiary of Honeywell International, Inc., for the U.S. Department of Energy's National Nuclear Security Administration under Contract No. DE-NA0003525.

Z. Lin acknowledges support from the National Natural Science Foundation of China (Grant Nos. 11574284 and 11774324).

J. Garza thanks CONACYT for support under the Project No. FC-2016/2412.

K. Lopata gratefully acknowledges support by the U.S. Department of Energy, Office of Science, Basic Energy Sciences, Early Career Program, under Award No. DE-SC0017868 and the U.S. Department of Energy, Office of Science, Basic Energy Sciences, under Award No. DE-SC0012462.

L. Gagliardi and C. J. Cramer acknowledge support from by the Inorganometallic Catalyst Design Center, an Energy Frontier Research Center funded by the US Department of Energy (DOE), Office of Science, Basic Energy Sciences (BES) (DESC0012702). They acknowledge the Minnesota Supercomputing Institute (MSI) at the University of Minnesota for providing computational resources.

This work used resources provided by EMSL, a DOE Office of Science User Facility sponsored by the Office of Biological and Environmental Research and located at the Pacific Northwest National Laboratory (PNNL) and PNNL Institutional Computing (PIC). PNNL is operated by Battelle Memorial Institute for the United States Department of Energy under DOE Contract No. DE-AC0576RL1830.

The work also used resources provided by the National Energy Research Scientific Computing Center (NERSC), a DOE Office of Science User Facility supported by the Office of Science of the U.S. Department of Energy under Contract No. DE-AC02-05CH11231.

This work also used resources provided by the Oak Ridge Leadership Computing Facility (OLCF) at the Oak Ridge National Laboratory, which is supported by the Office of Science of the U.S. Department of Energy under Contract No. DE-AC05-00OR22725.

This manuscript was authored, in part, by UT-Battelle, LLC, under Contract No. DE-AC05-00OR22725 with the U.S. Department of Energy (DOE). The U.S. government retains and the publisher, by accepting the article for publication, acknowledges that the U.S. government retains a nonexclusive, paid-up, irrevocable, worldwide license to publish or reproduce the published form of this manuscript, or allow others to do so, for U.S. government purposes. The DOE will provide public access to these results of federally sponsored research in accordance with the DOE Public Access Plan (http://energy.gov/downloads/doe-public-access-plan).

\section{DATA AVAILABILITY}

The data that support the findings of this study are available from the corresponding author upon reasonable request.

\section{REFERENCES}

${ }^{1}$ D. E. Bernholdt, E. Apra, H. A. Früchtl, M. F. Guest, R. J. Harrison, R. A. Kendall, R. A. Kutteh, X. Long, J. B. Nicholas, J. A. Nichols, H. L. Taylor, A. T. Wong, G. I. Fann, R. J. Littlefield, and J. Nieplocha, Int. J. Quantum Chem. 56, 475 (1995).

${ }^{2}$ R. A. Kendall, E. Aprà, D. E. Bernholdt, E. J. Bylaska, M. Dupuis, G. I. Fann, R. J. Harrison, J. Ju, J. A. Nichols, J. Nieplocha, T. P. Straatsma, T. L. Windus, and A. T. Wong, Comput. Phys. Commun. 128, 260 (2000).
${ }^{3}$ M. Valiev, E. J. Bylaska, N. Govind, K. Kowalski, T. P. Straatsma, H. J. J. Van Dam, D. Wang, J. Nieplocha, E. Apra, T. L. Windus, and W. A. de Jong, Comput. Phys. Commun. 181, 1477 (2010).

${ }^{4}$ R. J. Harrison, J. A. Nichols, T. P. Straatsma, M. Dupuis, E. J. Bylaska, G. I. Fann, T. L. Windus, E. Apra, J. Anchell, D. Bernholdt, P. Borowski, T. Clark, D. Clerc, H. Dachsel, W. A. de Jong, M. Deegan, K. Dyall, D. Elwood, H. Fruchtl, E. Glendening, M. Gutowski, A. Hess, J. Jaffe, B. Johnson, J. Ju, R. Kendall, R. Kobayashi, R. Kutteh, Z. Lin, R. Littlefield, X. Long, B. Meng, J. Nieplocha, A. Rendall, M. Rosing, G. Sandrone, M. Stave, H. Taylor, G. Thomas, J. van Lenthe, K. Wolinski, A. Wong, and Z. Zhang, NWChem, A Computational Chemistry Package for Parallel Computers, version 4.1 (Pacific Northwest National Laboratory, Richland, WA, 2000).

${ }^{5}$ T. P. Straatsma, E. Apra, T. Windus, E. Bylaska, W. de Jong, S. Hirata, M. Valiev, M. Hackler, L. Pollack, R. Harrison, M. Dupuis, D. Smith, J. Nieplocha, V. Tipparaju, M. Krishnan, A. Auer, E. Brown, G. Cisneros, G. Fann, H. Fruchtl, J. Garza, K. Hirao, R. Kendall, J. Nichols, K. Tsemekhman, K. Wolinski, J. Anchell, D. Bernholdt, P. Borowski, T. Clark, D. Clerc, H. Dachsel, M. Deegan, K. Dyall, D. Elwood, E. Glendening, M. Gutowski, A. Hess, J. Jaffe, B. Johnson, J. Ju, R. Kobayashi, R. Kutteh, Z. Lin, R. Littlefield, X. Long, B. Meng, T. Nakajima, S. Niu, M. Rosing, G. Sandrone, M. Stave, H. Taylor, G. Thomas, J. van Lenthe, A. Wong, and Z. Zhang, NWChem, A Computational Chemistry Package for Parallel Computers, Version 4.6 (2004) (Pacific Northwest National Laboratory, Richland, WA, 2004). ${ }^{6}$ E. Apra, T. Windus, T. P. Straatsma, E. Bylaska, W. de Jong, S. Hirata, M. Valiev, M. Hackler, L. Pollack, K. Kowalski, R. Harrison, M. Dupuis, D. Smith, J. Nieplocha, V. Tipparaju, M. Krishnan, A. Auer, E. Brown, G. Cisneros, G. Fann, H. Fruchtl, J. Garza, K. Hirao, R. Kendall, J. Nichols, K. Tsemekhman, K. Wolinski, J. Anchell, D. Bernholdt, P. Borowski, T. Clark, D. Clerc, H. Dachsel, M. Deegan, K. Dyall, D. Elwood, E. Glendening, M. Gutowski, A. Hess, J. Jaffe, B. Johnson, J. Ju, R. Kobayashi, R. Kutteh, Z. Lin, R. Littlefield, X. Long, B. Meng, T. Nakajima, S. Niu, M. Rosing, G. Sandrone, M. Stave, H. Taylor, G. Thomas, J. van Lenthe, A. Wong, and Z. Zhang, NWChem, A Computational Chemistry Package for Parallel Computers, Version 4.7 (Pacific Northwest National Laboratory, Richland, WA, 2005).

${ }^{7}$ E. J. Bylaska, W. A. de Jong, N. Govind, K. Kowalski, T. P. Straatsma, M. Valiev, D. Wang, E. Apra, T. L. Windus, J. Hammond, M. Hackler, Y. Zhao, R. Harrison, M. Dupuis, D. M. A. Smith, A. A. Auer, E. Brown, G. Cisneros, G. I. Fann, H. Fruchtl, J. Garza, K. Hirao, R. Kendall, J. A. Nichols, K. Tsemekhman, K. Wolinski, J. Anchell, D. Bernholdt, P. Borowski, T. Clark, D. Clerc, H. Dachsel, M. Deegan, K. Dyall, D. Elwood, E. Glendening, M. Gutowski, A. Hess, J. Jaffe, B. Johnson, J. Ju, R. Kobayashi, R. Kutteh, Z. Lin, R. Littlefield, X. Long, B. Meng, T. Nakajima, S. Niu, L. Pollack, M. Rosing, G. Sandrone, M. Stave, H. Taylor, G. Thomas, J. van Lenthe, A. Wong, and Z. Zhang, NWChem, A Computational Chemistry Package for Parallel Computers, Version 5.1 (Pacific Northwest National Laboratory, Richland, WA, 2007).

${ }^{8}$ See https:/github.com/nwchemgit/nwchem/wiki for NWChem-HighPerformance Computational Chemistry Software, 2019, online; accessed 29 August 2019.

${ }^{9}$ A. Szabo and N. S. Ostlund, Modern Quantum Chemistry: Introduction to Advanced Electronic Structure Theory (Courier Corporation, 2012).

${ }^{10} \mathrm{C}$. J. Cramer, Essentials of Computational Chemistry: Theories and Models (John Wiley \& Sons, 2013).

${ }^{11}$ F. Jensen, Introduction to Computational Chemistry (John Wiley \& Sons, 2017).

${ }^{12}$ V. Fock, Z. Phys. 61, 126 (1930).

${ }^{13}$ J. C. Slater, Phys. Rev. 35, 210 (1930).

${ }^{14}$ V. Fock, Z. Phys. 62, 795 (1930).

${ }^{15}$ D. R. Hartree and W. Hartree, Proc. R. Soc. London, Ser. A 150, 9 (1935).

${ }^{16}$ P. Hohenberg and W. Kohn, Phys. Rev. 136, B864 (1964).

${ }^{17}$ W. Kohn and L. J. Sham, Phys. Rev. 140, A1133 (1965).

${ }^{18}$ R. G. Parr and W. Yang, Density-Functional Theory of Atoms and Molecules, International Series of Monographs on Chemistry (Oxford University Press, USA, 1994).

${ }^{19}$ R. Car and M. Parrinello, Phys. Rev. Lett. 55, 2471 (1985).

${ }^{20}$ D. R. Hamann, Phys. Rev. B 40, 2980 (1989).

${ }^{21}$ N. Troullier and J. L. Martins, Phys. Rev. B 43, 1993 (1991).

${ }^{22}$ G. B. Bachelet, D. R. Hamann, and M. Schlüter, Phys. Rev. B 26, 4199 (1982). 
${ }^{23}$ P. E. Blöchl, Phys. Rev. B 50, 17953 (1994).

${ }^{24}$ J. Anchell, E. Apra, D. Bernholdt, P. Borowski, T. Clark, D. Clerc, H. Dachsel, M. Deegan, M. Dupuis, K. Dyall, G. Fann, H. Fruchtl, M. Gutowski, R. Harrison, A. Hess, J. Jaffe, R. Kendall, R. Kobayashi, R. Kutteh, Z. Lin, R. Littlefield, X. Long, B. Meng, J. Nichols, J. Nieplocha, A. Rendall, M. Stave, T. P. Straatsma, H. Taylor, G. Thomas, K. Wolinski, and A. Wong, NWChem, Version 3.2, High Performance Computational Chemistry Group (Pacific Northwest National Laboratory, Richland, WA, 1998).

${ }^{25}$ T. P. Straatsma, E. J. Bylaska, H. J. J. van Dam, N. Govind, W. A. de Jong, K. Kowalski, and M. Valiev, Annu. Rep. Comput. Chem. 7, 151 (2011).

${ }^{26}$ E. Aprà, A. P. Rendell, R. J. Harrison, V. Tipparaju, W. A. de Jong, and S. S. Xantheas, in Proceedings of the Conference on High Performance Computing Networking, Storage and Analysis (ACM, 2009), p. 66.

${ }^{27} \mathrm{~V}$. Tipparaju, E. Aprà, W. Yu, and J. S. Vetter, in Proceedings of the 7th ACM International Conference on Computing Frontiers (ACM, 2010), pp. 207-216.

${ }^{28} \mathrm{~K}$. Kowalski, S. Krishnamoorthy, R. M. Olson, V. Tipparaju, and E. Apra, in Proceedings of 2011 International Conference for High Performance Computing, Networking, Storage and Analysis (ACM, 2011), p. 72.

${ }^{29}$ E. J. Bylaska, K. Tsemekhman, N. Govind, and M. Valiev, in Computational Methods for Large Systems: Electronic Structure Approaches for Biotechnology and Nanotechnology, edited by J. R. Reimers (Wiley Online Library, 2011), pp. 77-116. ${ }^{30}$ G. D. Purvis and R. J. Bartlett, J. Chem. Phys. 76, 1910 (1982).

${ }^{31}$ K. Raghavachari, G. W. Trucks, J. A. Pople, and M. Head-Gordon, Chem. Phys. Lett. 157, 479 (1989).

${ }^{32}$ W. Ma, S. Krishnamoorthy, O. Villa, and K. Kowalski, J. Chem. Theory Comput. 7, 1316 (2011)

${ }^{33}$ E. J. Bylaska, M. Jacquelin, W. A. de Jong, J. Hammond, and M. Klemm, in High Performance Computing, edited by J. M. Kunkel, R. Yokota, M. Taufer, and J. Shalf (Springer International Publishing, Cham, 2017), pp. 404-418.

${ }^{34}$ E. J. Bylaska, E. Aprà, K. Kowalski, M. Jacquelin, W. A. de Jong, A. Vishnu, B. Palmer, J. Daily, T. P. Straatsma, and J. R. Hammond, Exascale Scientific Applications: Scalability and Performance Portability (CRC Press, 2017), p. 165.

${ }^{35} \mathrm{E}$. Apra, M. Klemm, and K. Kowalski, in Proceedings of the International Conference for High Performance Computing, Networking, Storage and Analysis (IEEE Press, 2014), pp. 674-684.

${ }^{36}$ J. Nieplocha, R. Harrison, and R. Littlefield, SIAM News 28, 12 (1995).

${ }^{37}$ J. Nieplocha, R. J. Harrison, and R. J. Littlefield, J. Supercomput. 10, 169 (1996).

${ }^{38}$ J. Nieplocha, B. Palmer, V. Tipparaju, M. Krishnan, H. Trease, and E. Aprà, Int. J. High Perform. Comput. Appl. 20, 203 (2006).

${ }^{39}$ M. Krishnan, B. Palmer, A. Vishnu, S. Krishnamoorthy, J. Daily, and D. Chavarria, The Global Arrays User Manual (Pacific Northwest National Laboratory, Richland, WA, 2012).

${ }^{40} \mathrm{~J}$. Nieplocha, V. Tipparaju, M. Krishnan, and D. K. Panda, Int. J. High Perform. Comput. Appl. 20, 233 (2006)

${ }^{41}$ J. Daily, A. Vishnu, B. Palmer, H. van Dam, and D. Kerbyson, in 2014 21st International Conference on High Performance Computing (HiPC) (IEEE, 2014), pp. 1-10.

${ }^{42}$ J. Dinan, P. Balaji, J. R. Hammond, S. Krishnamoorthy, and V. Tipparaju, in 2012 IEEE 26th International Parallel and Distributed Processing Symposium (IEEE, 2012), pp. 739-750.

${ }^{43}$ A. T. Wong and R. J. Harrison, J. Comput. Chem. 16, 1291 (1995).

${ }^{44}$ I. T. Foster, J. L. Tilson, A. F. Wagner, R. L. Shepard, R. J. Harrison, R. A. Kendall, and R. J. Littlefield, J. Comput. Chem. 17, 109 (1996).

${ }^{45}$ R. A. Kendall and H. A. Früchtl, Theor. Chem. Acc. 97, 158 (1997).

${ }^{46} \mathrm{See}$ https://tinyurl.com/nwchem-xc for Summary table of XC functionals available in NWChem.

${ }^{47}$ B. I. Dunlap, J. W. D. Connolly, and J. R. Sabin, J. Chem. Phys. 71, 3396 (1979).

${ }^{48} \mathrm{~B}$. Palmer, J. Nieplocha, and E. Aprà, in 2003 Proceedings IEEE International Conference on Cluster Computing (IEEE, 2003), pp. 420-428.

${ }^{49}$ G. I. Fann and R. J. Littlefield, in Proceedings of the 6th SIAM Conference on Parallel Processing for Scientific Computing (SIAM, Philadelphia, PA, USA, 1993).

${ }^{50} \mathrm{G}$. Fann, R. Littlefield, and D. Elwood, in Proceedings of the High Performance Computing '95, Simulation MultiConference (Society for Computer Simulation, San Diego, CA, USA, 1995).
${ }^{51}$ I. S. Dhillon, G. Fann, and B. N. Parlett, in SIAM Conference on Parallel Processing for Scientific Computing (SIAM, 1997).

${ }^{52}$ L. S. Blackford, J. Choi, A. Cleary, E. D’Azeuedo, J. Demmel, I. Dhillon, S. Hammarling, G. Henry, A. Petitet, K. Stanley, D. Walker, and R. C. Whaley, in ScaLAPACK User's Guide, edited by J. J. Dongarra (Society for Industrial and Applied Mathematics, Philadelphia, PA, USA, 1997).

${ }^{53}$ T. Auckenthaler, V. Blum, H.-J. Bungartz, T. Huckle, R. Johanni, L. Krämer, B. Lang, H. Lederer, and P. R. Willems, Parallel Comput. 37, 783 (2011).

${ }^{54}$ A. Marek, V. Blum, R. Johanni, V. Havu, B. Lang, T. Auckenthaler, A. Heinecke, H.-J. Bungartz, and H. Lederer, J. Phys.: Condens. Matter 26, 213201 (2014).

${ }^{55}$ P. Pulay, Chem. Phys. Lett. 73, 393 (1980).

${ }^{56}$ V. R. Saunders and I. H. Hillier, Int. J. Quantum Chem. 7, 699 (1973).

${ }^{57}$ M. F. Guest and V. R. Saunders, Mol. Phys. 28, 819 (1974).

${ }^{58}$ R. W. Warren and B. I. Dunlap, Chem. Phys. Lett. 262, 384 (1996).

${ }^{59}$ S. Refaely-Abramson, S. Sharifzadeh, N. Govind, J. Autschbach, J. B. Neaton, R. Baer, and L. Kronik, Phys. Rev. Lett. 109, 226405 (2012).

${ }^{60}$ T. Stein, J. Autschbach, N. Govind, L. Kronik, and R. Baer, J. Phys. Chem. Lett. 3, 3740 (2012).

${ }^{61}$ M. Srebro and J. Autschbach, J. Chem. Theory Comput. 8, 245 (2012).

${ }^{62}$ B. Moore, M. Srebro, and J. Autschbach, J. Chem. Theory Comput. 8, 4336 (2012).

${ }^{63}$ J. Autschbach and M. Srebro, Acc. Chem. Res. 47, 2592 (2014).

${ }^{64} \mathrm{H}$. Sun and J. Autschbach, ChemPhysChem 14, 2450 (2013).

${ }^{65}$ H. Sun and J. Autschbach, J. Chem. Theory Comput. 10, 1035 (2014).

${ }^{66}$ B. Moore, H. Sun, N. Govind, K. Kowalski, and J. Autschbach, J. Chem. Theory Comput. 11, 3305 (2015).

${ }^{67}$ J. P. Perdew and A. Zunger, Phys. Rev. B 23, 5048 (1981).

${ }^{68}$ J. Garza, R. Vargas, J. A. Nichols, and D. A. Dixon, J. Chem. Phys. 114, 639 (2001).

${ }^{69}$ R. T. Sharp and G. K. Horton, Phys. Rev. 90, 317 (1953).

${ }^{70}$ J. D. Talman and W. F. Shadwick, Phys. Rev. A 14, 36 (1976).

${ }^{71}$ J. B. Krieger, Y. Li, and G. J. Iafrate, Phys. Rev. A 45, 101 (1992).

${ }^{72}$ Y. Li, J. B. Krieger, and G. J. Iafrate, Phys. Rev. A 47, 165 (1993).

${ }^{73}$ M. E. Casida and D. R. Salahub, J. Chem. Phys. 113, 8918 (2000).

${ }^{74}$ S. Hirata, C.-G. Zhan, E. Aprà, T. L. Windus, and D. A. Dixon, J. Phys. Chem. A 107, 10154 (2003).

${ }^{75}$ J. Carmona-Espíndola, J. L. Gázquez, A. Vela, and S. B. Trickey, J. Chem. Theory Comput. 15, 303 (2018).

${ }^{76}$ A. Otero-de-la-Roza and E. R. Johnson, J. Chem. Phys. 138, 204109 (2013).

${ }^{77}$ S. Grimme, J. Antony, S. Ehrlich, and H. Krieg, J. Chem. Phys. 132, 154104 (2010).

${ }^{78}$ S. Grimme, S. Ehrlich, and L. Goerigk, J. Comput. Chem. 32, 1456 (2011).

${ }^{79}$ Y. Zhao and D. G. Truhlar, Chem. Phys. Lett. 502, 1 (2011).

${ }^{80}$ A. D. Becke, J. Chem. Phys. 88, 2547 (1988).

${ }^{81}$ R. E. Stratmann, G. E. Scuseria, and M. J. Frisch, Chem. Phys. Lett. 257, 213 (1996).

${ }^{82}$ M. E. Mura and P. J. Knowles, J. Chem. Phys. 104, 9848 (1996).

${ }^{83}$ V. I. Lebedev and D. Laikov, Dokl. Math. 59, 477 (1999) [translated from Dokl. Akad. Nauk, 36(6), 741-745 (1999)].

${ }^{84}$ A. Farazdel, M. Dupuis, E. Clementi, and A. Aviram, J. Am. Chem. Soc. 112, 4206 (1990).

${ }^{85}$ K. M. Rosso, D. M. A. Smith, and M. Dupuis, J. Chem. Phys. 118, 6455 (2003).

${ }^{86} \mathrm{Q}$. Wu and T. Van Voorhis, Phys. Rev. A 72, 024502 (2005).

${ }^{87} \mathrm{Q}$. Wu and T. Van Voorhis, J. Chem. Theory Comput. 2, 765 (2006).

${ }^{88} \mathrm{Q}$. Wu and T. Van Voorhis, J. Phys. Chem. A 110, 9212 (2006).

${ }^{89}$ T. A. Wesolowski and A. Warshel, J. Phys. Chem. 97, 8050 (1993).

${ }^{90}$ T. A. Wesołowski, Phys. Rev. A 77, 012504 (2008).

${ }^{91}$ Y. Lu, M. R. Farrow, P. Fayon, A. J. Logsdail, A. A. Sokol, C. R. A. Catlow, P. Sherwood, and T. W. Keal, J. Chem. Theory Comput. 15, 1317 (2019).

${ }^{92}$ J. B. Foresman, M. Head-Gordon, J. A. Pople, and M. J. Frisch, J. Phys. Chem. 96, 135 (1992). 
${ }^{93}$ C. Jamorski, M. E. Casida, and D. R. Salahub, J. Chem. Phys. 104, 5134 (1996).

${ }^{94}$ R. Bauernschmitt and R. Ahlrichs, Chem. Phys. Lett. 256, 454 (1996).

${ }^{95}$ R. Bauernschmitt, M. Häser, O. Treutler, and R. Ahlrichs, Chem. Phys. Lett. 264, 573 (1997).

${ }^{96}$ S. Hirata and M. Head-Gordon, Chem. Phys. Lett. 314, 291 (1999).

${ }^{97}$ E. Vecharynski, J. Brabec, M. Shao, N. Govind, and C. Yang, Comput. Phys. Commun. 221, 42 (2017).

${ }^{98}$ K. Lopata, B. E. Van Kuiken, M. Khalil, and N. Govind, J. Chem. Theory Comput. 8, 3284 (2012).

${ }^{99}$ Y. Zhang, S. Mukamel, M. Khalil, and N. Govind, J. Chem. Theory Comput. 11, 5804 (2015)

${ }^{100}$ Y. Zhang, U. Bergmann, R. Schoenlein, M. Khalil, and N. Govind, J. Chem. Phys. 151, 144114 (2019).

${ }^{101}$ D. W. Silverstein, N. Govind, H. J. J. Van Dam, and L. Jensen, J. Chem. Theory Comput. 9, 5490 (2013).

${ }^{102}$ M. Srebro, N. Govind, W. A. de Jong, and J. Autschbach, J. Phys. Chem. A 115, 10930 (2011).

${ }^{103}$ J. Autschbach, ChemPhysChem 12, 3224 (2011).

${ }^{104}$ Y. Zhang, J. R. Rouxel, J. Autschbach, N. Govind, and S. Mukamel, Chem. Sci. 8, 5969 (2017).

${ }^{105}$ K. Lopata and N. Govind, J. Chem. Theory Comput. 7, 1344 (2011).

${ }^{106}$ S. Tussupbayev, N. Govind, K. Lopata, and C. J. Cramer, J. Chem. Theory Comput. 11, 1102 (2015).

${ }^{107}$ S. A. Fischer, C. J. Cramer, and N. Govind, J. Chem. Theory Comput. 11, 4294 (2015).

${ }^{108}$ D. N. Bowman, J. C. Asher, S. A. Fischer, C. J. Cramer, and N. Govind, Phys. Chem. Chem. Phys. 19, 27452 (2017).

${ }^{109}$ A. Bruner, S. Hernandez, F. Mauger, P. M. Abanador, D. J. LaMaster, M. B. Gaarde, K. J. Schafer, and K. Lopata, J. Phys. Chem. Lett. 8, 3991 (2017).

${ }^{110}$ D. Cho, J. R. Rouxel, M. Kowalewski, P. Saurabh, J. Y. Lee, and S. Mukamel, J. Phys. Chem. Lett. 9, 1072 (2018).

${ }^{111}$ A. Bruner, S. M. Cavaletto, N. Govind, and S. Mukamel, J. Chem. Theory Comput. 15, 6832 (2019).

${ }^{112}$ Y. Wang, K. Lopata, S. A. Chambers, N. Govind, and P. V. Sushko, J. Phys. Chem. C 117, 25504 (2013).

113 A. Castro, M. A. L. Marques, and A. Rubio, J. Chem. Phys. 121, 3425 (2004).

${ }^{114}$ W. Magnus, Commun. Pure Appl. Math. 7, 649 (1954).

${ }^{115}$ L. Verlet, Phys. Rev. 159, 98 (1967).

${ }^{116}$ W. C. Swope, H. C. Andersen, P. H. Berens, and K. R. Wilson, J. Chem. Phys. 76, 637 (1982)

${ }^{117}$ G. Bussi, D. Donadio, and M. Parrinello, J. Chem. Phys. 126, 014101 (2007).

${ }^{118}$ G. Bussi and M. Parrinello, Phys. Rev. E 75, 056707 (2007).

${ }^{119}$ H. J. C. Berendsen, J. P. M. Postma, W. F. van Gunsteren, A. DiNola, and J. R. Haak, J. Chem. Phys. 81, 3684 (1984).

${ }^{120}$ S. A. Haak, T. W. Ueltschi, P. Z. El-Khoury, A. L. Mifflin, W. P. Hess, H.-F. Wang, C. J. Cramer, and N. Govind, J. Phys. Chem. B 120, 1429 (2016).

${ }^{121}$ I. Lindgren and J. Morrison, Atomic Many-Body Theory (Springer Science \& Business Media, 2012), Vol. 3.

${ }^{122}$ I. Shavitt and R. J. Bartlett, Many-Body Methods in Chemistry and Physics: MBPT and Coupled-Cluster Theory (Cambridge University Press, 2009).

${ }^{123}$ C. Møller and M. S. Plesset, Phys. Rev. 46, 618 (1934).

${ }^{124}$ A. T. Wong, R. J. Harrison, and A. P. Rendell, Theor. Chim. Acta 93, 317 (1996).

${ }^{125}$ D. E. Bernholdt and R. J. Harrison, Chem. Phys. Lett. 250, 477 (1996).

${ }^{126}$ J. Olsen, P. Jørgensen, and J. Simons, Chem. Phys. Lett. 169, 463 (1990).

${ }^{127}$ K. D. Vogiatzis, D. Ma, J. Olsen, L. Gagliardi, and W. A. de Jong, J. Chem. Phys. 147, 184111 (2017).

${ }^{128}$ S. Hirata, J. Phys. Chem. A 107, 9887 (2003).

${ }^{129}$ G. Baumgartner, A. Auer, D. E. Bernholdt, A. Bibireata, V. Choppella, D. Cociorva, X. Gao, R. J. Harrison, S. Hirata, S. Krishnamoorthy, S. Krishnan, C.-chung Lam, Q. Lu, M. Nooijen, R. M. Pitzer, J. Ramanujam, P. Sadayappan, and A. Sibiryakov, Proc. IEEE 93, 276 (2005).
${ }^{130}$ J. Čížek, J. Chem. Phys. 45, 4256 (1966).

${ }^{131}$ J. Čížek and J. Paldus, Int. J. Quantum Chem. 5, 359 (1971).

${ }^{132}$ R. Kobayashi and A. P. Rendell, Chem. Phys. Lett. 265, 1 (1997).

${ }^{133}$ G. E. Scuseria, C. L. Janssen, and H. F. Schaefer, J. Chem. Phys. 89, 7382 (1988).

${ }^{134}$ T. J. Lee, A. P. Rendell, and J. E. Rice, TITAN-A set of electronic structure programs.

${ }^{135}$ A. P. Rendell, T. J. Lee, A. Komornicki, and S. Wilson, Theor. Chim. Acta 84, 271 (1993).

${ }^{136}$ S. Yoo, E. Aprà, X. C. Zeng, and S. S. Xantheas, J. Phys. Chem. Lett. 1, 3122 (2010).

${ }^{137}$ V. M. Anisimov, G. H. Bauer, K. Chadalavada, R. M. Olson, J. W. Glenski, W. T. C. Kramer, E. Aprà, and K. Kowalski, J. Chem. Theory Comput. 10, 4307 (2014).

${ }^{138}$ S. Hirata, Theor. Chem. Acc. 116, 2 (2006).

${ }^{139}$ C. L. Janssen and H. F. Schaefer, Theor. Chim. Acta 79, 1 (1991).

${ }^{140} \mathrm{X}$. Li and J. Paldus, J. Chem. Phys. 101, 8812 (1994).

${ }^{141}$ M. Nooijen and V. Lotrich, J. Mol. Struct.: THEOCHEM 547, 253 (2001).

${ }^{142}$ M. Nooijen, Int. J. Mol. Sci. 3, 656 (2002).

${ }^{143}$ J. A. Parkhill, K. Lawler, and M. Head-Gordon, J. Chem. Phys. 130, 084101 (2009).

${ }^{144}$ E. Deumens, V. F. Lotrich, A. Perera, M. J. Ponton, B. A. Sanders, and R. J. Bartlett, Wiley Interdiscip. Rev.: Comput. Mol. Sci. 1, 895 (2011).

${ }^{145}$ M. K. MacLeod and T. Shiozaki, J. Chem. Phys. 142, 051103 (2015).

${ }^{146}$ S. Hirata, J. Chem. Phys. 121, 51 (2004).

${ }^{147}$ J. Noga and R. J. Bartlett, J. Chem. Phys. 86, 7041 (1987).

${ }^{148}$ J. Noga and R. J. Bartlett, J. Chem. Phys. 89, 3401 (1988).

${ }^{149}$ G. E. Scuseria and H. F. Schaefer, Chem. Phys. Lett. 152, 382 (1988).

${ }^{150} \mathrm{~N}$. Oliphant and L. Adamowicz, J. Chem. Phys. 95, 6645 (1991).

${ }^{151}$ S. A. Kucharski and R. J. Bartlett, Theor. Chem. Acc. 80, 387 (1991).

${ }^{152}$ K. Kowalski and P. Piecuch, J. Chem. Phys. 113, 18 (2000).

${ }^{153}$ K. Kowalski and P. Piecuch, J. Chem. Phys. 122, 074107 (2005).

${ }^{154}$ S. R. Gwaltney and M. Head-Gordon, Chem. Phys. Lett. 323, 21 (2000).

${ }^{155}$ S. R. Gwaltney and M. Head-Gordon, J. Chem. Phys. 115, 2014 (2001).

${ }^{156}$ S. Hirata, P.-D. Fan, A. A. Auer, M. Nooijen, and P. Piecuch, J. Chem. Phys. 121, 12197 (2004).

${ }^{157}$ J. Geertsen, M. Rittby, and R. J. Bartlett, Chem. Phys. Lett. 164, 57 (1989).

${ }^{158}$ D. C. Comeau and R. J. Bartlett, Chem. Phys. Lett. 207, 414 (1993).

${ }^{159}$ K. Kowalski and P. Piecuch, J. Chem. Phys. 115, 643 (2001).

${ }^{160}$ S. A. Kucharski, M. Włoch, M. Musiał, and R. J. Bartlett, J. Chem. Phys. 115, 8263 (2001).

${ }^{161}$ M. Kállay and J. Gauss, J. Chem. Phys. 121, 9257 (2004).

${ }^{162}$ K. Kowalski and P. Piecuch, J. Chem. Phys. 120, 1715 (2004).

${ }^{163}$ J. F. Stanton and R. J. Bartlett, J. Chem. Phys. 98, 7029 (1993).

${ }^{164}$ M. Nooijen and R. J. Bartlett, J. Chem. Phys. 102, 3629 (1995).

${ }^{165}$ J. F. Stanton and J. Gauss, J. Chem. Phys. 103, 1064 (1995).

${ }^{166} \mathrm{M}$. Musiał and R. J. Bartlett, J. Chem. Phys. 119, 1901 (2003).

${ }^{167}$ M. Musiał, S. A. Kucharski, and R. J. Bartlett, J. Chem. Phys. 118, 1128 (2003).

${ }^{168}$ J. R. Gour and P. Piecuch, J. Chem. Phys. 125, 234107 (2006).

${ }^{169}$ M. Kamiya and S. Hirata, J. Chem. Phys. 125, 074111 (2006).

${ }^{170}$ M. Kamiya and S. Hirata, J. Chem. Phys. 126, 134112 (2007).

${ }^{171}$ H. Koch and P. Jørgensen, J. Chem. Phys. 93, 3333 (1990).

${ }^{172}$ J. Pittner, P. Nachtigall, P. Čársky, J. Mášik, and I. Hubač, J. Chem. Phys. 110, 10275 (1999).

${ }^{173}$ J. Pittner, J. Chem. Phys. 118, 10876 (2003).

${ }^{174}$ U. S. Mahapatra, B. Datta, and D. Mukherjee, Mol. Phys. 94, 157 (1998).

${ }^{175}$ U. S. Mahapatra, B. Datta, B. Bandyopadhyay, and D. Mukherjee, State-Specific Multi-Reference Coupled Cluster Formulations: Two Paradigms (Academic Press, 1998), pp. 163-193.

${ }^{176}$ U. S. Mahapatra, B. Datta, and D. Mukherjee, J. Chem. Phys. 110, 6171 (1999).

${ }^{177}$ F. A. Evangelista, W. D. Allen, and H. F. Schaefer III, J. Chem. Phys. 127, 024102 (2007). 
${ }^{178}$ F. A. Evangelista, A. C. Simmonett, W. D. Allen, H. F. Schaefer III, and J. Gauss, J. Chem. Phys. 128, 124104 (2008).

${ }^{179}$ K. Bhaskaran-Nair, W. Ma, S. Krishnamoorthy, O. Villa, H. J. J. van Dam, E. Aprà, and K. Kowalski, J. Chem. Theory Comput. 9, 1949 (2013).

${ }^{180}$ E. Aprà and K. Kowalski, J. Chem. Theory Comput. 12, 1129 (2016).

${ }^{181}$ J. Brabec, J. Pittner, H. J. J. van Dam, E. Aprà, and K. Kowalski, J. Chem. Theory Comput. 8, 487 (2012).

${ }^{182}$ K. Bhaskaran-Nair, J. Brabec, E. Aprà, H. J. J. van Dam, J. Pittner, and K. Kowalski, J. Chem. Phys. 137, 094112 (2012).

${ }^{183}$ K. Kowalski, J. R. Hammond, W. A. de Jong, and A. J. Sadlej, J. Chem. Phys. 129, 226101 (2008).

${ }^{184}$ K. Kowalski, R. M. Olson, S. Krishnamoorthy, V. Tipparaju, and E. Aprà, J. Chem. Theory Comput. 7, 2200 (2011).

${ }^{185}$ M. Nooijen and J. G. Snijders, Int. J. Quantum Chem. 44, 55 (1992).

${ }^{186}$ M. Nooijen and J. G. Snijders, Int. J. Quantum Chem. 48, 15 (1993).

${ }^{187}$ M. Nooijen and J. G. Snijders, J. Chem. Phys. 102, 1681 (1995).

${ }^{188}$ L. Meissner and R. J. Bartlett, Int. J. Quantum Chem. 48, 67 (1993).

${ }^{189}$ E. Deumens, V. F. Lotrich, A. S. Perera, R. J. Bartlett, N. Jindal, and B. A. Sanders, Ann. Rep. Comput. Chem. 7, 179 (2011).

${ }^{190}$ E. Solomonik, D. Matthews, J. R. Hammond, J. F. Stanton, and J. Demmel, J Parallel Distrib. Comput. 74, 3176 (2014).

${ }^{191}$ C. Peng, J. A. Calvin, F. Pavošević, J. Zhang, and E. F. Valeev, J. Phys. Chem. A 120, 10231 (2016).

${ }^{192}$ E. Epifanovsky, M. Wormit, T. Kuś, A. Landau, D. Zuev, K. Khistyaev, P. Manohar, I. Kaliman, A. Dreuw, and A. I. Krylov, J. Comput. Chem. 34, 2293 (2013).

${ }^{193}$ P. A. M. Dirac, Proc. R. Soc. London, Ser. A 117, 610 (1928), containing Papers of a Mathematical and Physical Character.

${ }^{194}$ K. G. Dyall and K. Fægri, Jr., Introduction to Relativistic Quantum Chemistry (Oxford University Press, 2007).

${ }^{195}$ T. Saue, ChemPhysChem 12, 3077 (2011).

${ }^{196}$ M. Reiher and A. Wolf, Relativistic Quantum Chemistry, 2nd ed. (Wiley VCH, 2015).

${ }^{197}$ J. Autschbach, N. Govind, R. Atta-Fynn, E. J. Bylaska, J. W. Weare, and W. A. de Jong, "Computational tools for predictive modeling of properties in complex actinide systems," in Computational Methods in Lanthanide and Actinide Chemistry, edited by M. Dolg (John Wiley \& Sons, Ltd., 2015), Chap. 12, pp. 299-342.

${ }^{198}$ M. Douglas and N. M. Kroll, Ann. Phys. 82, 89 (1974).

${ }^{199}$ B. A. Hess, Phys. Rev. A 32, 756 (1985).

${ }^{200}$ B. A. Hess, Phys. Rev. A 33, 3742 (1986).

${ }^{201}$ C. Chang, M. Pelissier, and P. Durand, Phys. Scr. 34, 394 (1986).

${ }^{202}$ E. van Lenthe, "The ZORA equation," Ph.D. thesis, University of Amsterdam, 1996.

${ }^{203}$ S. Faas, J. G. Snijders, J. H. van Lenthe, E. van Lenthe, and E. J. Baerends, Chem. Phys. Lett. 246, 632 (1995).

${ }^{204}$ P. Nichols, N. Govind, E. J. Bylaska, and W. A. de Jong, J. Chem. Theory Comput. 5, 491 (2009).

${ }^{205}$ K. G. Dyall, J. Chem. Phys. 100, 2118 (1994).

${ }^{206}$ K. G. Dyall, J. Chem. Phys. 106, 9618 (1997).

${ }^{207}$ K. G. Dyall and T. Enevoldsen, J. Chem. Phys. 111, 10000 (1999).

${ }^{208}$ D. Peng and M. Reiher, Theor. Chem. Acc. 131, 1081 (2012).

${ }^{209}$ J. Autschbach, D. Peng, and M. Reiher, J. Chem. Theory Comput. 8, 4239 (2012).

${ }^{210}$ J. Autschbach, Philos. Trans. R. Soc., A 372, 20120489 (2014).

${ }^{211}$ J. Autschbach, J. Chem. Theory Comput. 13, 710 (2017).

${ }^{212}$ O. D. Häberlen and N. Rösch, Chem. Phys. Lett. 199, 491 (1992).

${ }^{213}$ T. Nakajima and K. Hirao, Chem. Phys. Lett. 329, 511 (2000).

${ }^{214}$ T. Nakajima and K. Hirao, J. Chem. Phys. 113, 7786 (2000).

${ }^{215}$ J. H. van Lenthe, S. Faas, and J. G. Snijders, Chem. Phys. Lett. 328, 107 (2000).

${ }^{216}$ J. H. van Lenthe and J. N. J. Van Lingen, Int. J. Quantum Chem. 106, 2525 (2006).
${ }^{217}$ C. van Wüllen, J. Chem. Phys. 109, 392 (1998).

${ }^{218}$ C. van Wüllen and C. Michauk, J. Chem. Phys. 123, 204113 (2005).

${ }^{219}$ J. M. Mullin, J. Autschbach, and G. C. Schatz, Comput. Theor. Chem. 987, 32 (2012).

${ }^{220}$ F. W. Aquino and G. C. Schatz, J. Phys. Chem. A 118, 517 (2014).

${ }^{221}$ F. Aquino, N. Govind, and J. Autschbach, J. Chem. Theory Comput. 6, 2669 (2010).

${ }^{222}$ R. Ditchfield, Mol. Phys. 27, 789 (1974).

${ }^{223}$ M. Dupuis, Comput. Phys. Commun. 134, 150 (2001).

${ }^{224}$ P. Verma and J. Autschbach, J. Chem. Theory Comput. 9, 1932 (2013).

${ }^{225} \mathrm{~F}$. Aquino, N. Govind, and J. Autschbach, J. Chem. Theory Comput. 7, 32783292 (2011).

${ }^{226}$ J. Autschbach, S. Patchkovskii, and B. Pritchard, J. Chem. Theory Comput. 7, 2175 (2011).

${ }^{227}$ F. Aquino, B. Pritchard, and J. Autschbach, J. Chem. Theory Comput. 8, 598 (2012).

${ }^{228}$ C. Shen, M. Srebro-Hooper, T. Weymuth, F. Krausbeck, J. T. L. Navarrete, F. J. Ramírez, B. Nieto-Ortega, J. Casado, M. Reiher, J. Autschbach et al., Chem. - Eur. J. 24, 15067 (2018).

${ }^{229}$ F. Krausbeck, J. Autschbach, and M. Reiher, J. Phys. Chem. A 120, 9740 (2016).

${ }^{230}$ J. R. Hammond, N. Govind, K. Kowalski, J. Autschbach, and S. S. Xantheas, J. Chem. Phys. 131, 214103 (2009).

${ }^{231}$ M. Jacquelin, W. A. de Jong, and E. J. Bylaska, in 2017 IEEE International Parallel and Distributed Processing Symposium (IPDPS) (IEEE Computer Society, 2017), pp. 234-243.

${ }^{232}$ E. J. Bylaska, M. Valiev, R. Kawai, and J. H. Weare, Comput. Phys. Commun. 143, 11 (2002).

${ }^{233}$ E. J. Bylaska, K. Tsemekhman, S. B. Baden, J. H. Weare, and H. Jonsson, J. Comput. Chem. 32, 54 (2011).

${ }^{234}$ E. J. Bylaska, Annual Reports in Computational Chemistry (Elsevier, 2017), Vol. 13, pp. $185-228$.

${ }^{235}$ M. Valiev, E. J. Bylaska, A. Gramada, and J. H. Weare, in Reviews in Modern Quantum Chemistry: A Celebration of the Contributions of Robert G. Parr, edited by K. D. Sen (World Scientific, Singapore, 2002).

${ }^{236}$ P. E. Blöchl and M. Parrinello, Phys. Rev. B 45, 9413 (1992).

${ }^{237}$ E. Cauët, S. A. Bogatko, E. J. Bylaska, and J. H. Weare, Inorg. Chem. 51, 10856 (2012).

${ }^{238}$ B. Roux, Comput. Phys. Commun. 91, 275 (1995).

${ }^{239}$ G. Bussi, A. Laio, and M. Parrinello, Phys. Rev. Lett. 96, 090601 (2006).

${ }^{240}$ A. Barducci, M. Bonomi, and M. Parrinello, Wiley Interdiscip. Rev.: Comput. Mol. Sci. 1, 826 (2011).

${ }^{241}$ L. Maragliano and E. Vanden-Eijnden, J. Chem. Phys. 128, 184110 (2008).

${ }^{242}$ L. Maragliano and E. Vanden-Eijnden, Chem. Phys. Lett. 426, 168 (2006).

${ }^{243}$ S. Kumar, J. M. Rosenberg, D. Bouzida, R. H. Swendsen, and P. A. Kollman, J. Comput. Chem. 13, 1011 (1992).

${ }^{244}$ J. J. Rehr and A. L. Ankudinov, J. Synchrotron Radiat. 8, 61 (2001).

${ }^{245}$ J. J. Rehr and R. C. Albers, Phys. Rev. B 41, 8139 (1990).

${ }^{246}$ A. L. Ankudinov, B. Ravel, J. J. Rehr, and S. D. Conradson, Phys. Rev. B 58, 7565 (1998).

${ }^{247}$ E. J. Bylaska and K. Rosso, J. Chem. Theory Comput. 14, 4416 (2018).

${ }^{248}$ P. L. Silvestrelli, Phys. Rev. B 59, 9703 (1999).

${ }^{249}$ A. Damle, L. Lin, and L. Ying, J. Chem. Theory Comput. 11, 1463 (2015).

${ }^{250}$ R. Kawai and J. H. Weare, J. Chem. Phys. 95, 1151 (1991).

${ }^{251}$ P. E. Blöchl, J. Chem. Phys. 103, 7422 (1995).

${ }^{252}$ V. Kapil, M. Rossi, O. Marsalek, R. Petraglia, Y. Litman, T. Spura, B. Cheng, A. Cuzzocrea, R. H. Meißner, D. M. Wilkins et al., Comput. Phys. Commun. 236, 214 (2019).

${ }^{253}$ A. H. Larsen, J. J. Mortensen, J. Blomqvist, I. E. Castelli, R. Christensen, M. Dułak, J. Friis, M. N. Groves, B. Hammer, C. Hargus, E. D. Hermes, P. C. Jennings, P. B. Jensen, J. Kermode, J. R. Kitchin, E. L. Kolsbjerg, J. Kubal, K. Kaasbjerg, S. Lysgaard, J. B. Maronsson, T. Maxson, T. Olsen, L. Pastewka, A. Peterson, C. Rostgaard, J. Schiøtz, O. Schütt, M. Strange, K. S. Thygesen, T. Vegge, 
L. Vilhelmsen, M. Walter, Z. Zeng, and K. W. Jacobsen, J. Phys.: Condens. Matter 29, 273002 (2017).

${ }^{254}$ G. Klimeck, M. McLennan, S. P. Brophy, G. B. Adams III, and M. S. Lundstrom, Comput. Sci. Eng. 10, 17 (2008).

${ }^{255}$ E. J. Bylaska, EMSL Arrows, https://arrows.emsl.pnnl.gov/api, 2019, online; accessed 6 December 2019.

${ }^{256}$ E. J. Bylaska, K. Tsemekhman, and F. Gao, Phys. Scr. T124, 86 (2006).

${ }^{257}$ D. C. Langreth, M. Dion, H. Rydberg, E. Schröder, P. Hyldgaard, and B. I. Lundqvist, Int. J. Quantum Chem. 101, 599 (2005).

${ }^{258}$ C. Hartwigsen, S. Goedecker, and J. Hutter, Phys. Rev. B 58, 3641 (1998).

${ }^{259}$ S. Goedecker, M. Teter, and J. Hutter, Phys. Rev. B 54, 1703 (1996).

${ }^{260}$ D. Hamann, Phys. Rev. B 88, 085117 (2013).

${ }^{261}$ M. Schlipf and F. Gygi, Comput. Phys. Commun. 196, 36 (2015).

${ }^{262}$ R. G. Parr and W. Yang, Horizons of Quantum Chemistry (Oxford University Press, 1994)

${ }^{263}$ W. E. Pickett, Rev. Mod. Phys. 61, 433 (1989).

${ }^{264}$ J. Ihm, A. Zunger, and M. L. Cohen, J. Phys. C: Solid State Phys. 12, 4409 (1979).

${ }^{265}$ M. C. Payne, M. P. Teter, D. C. Allan, T. A. Arias, and J. D. Joannopoulos, Rev. Mod. Phys. 64, 1045 (1992).

${ }^{266}$ D. K. Remler and P. A. Madden, Mol. Phys. 70, 921 (1990).

${ }^{267} \mathrm{G}$. Kresse and J. Furthmüller, Phys. Rev. B 54, 11169 (1996).

${ }^{268}$ D. Marx and J. Hutter, "Ab initio molecular dynamics: Theory and implementation," in Modern Methods and Algorithms of Quantum Chemistry, Vol. 1, edited by J. Grotendorst (NIC, 2000), pp. 301-449.

${ }^{269}$ R. M. Martin, Electronic Structure: Basic Theory and Practical Methods (Cambridge University Press, 2004).

${ }^{270}$ Y. Chen, E. J. Bylaska, and J. H. Weare, in Molecular Modeling of Geochemical Reactions: An Introduction, edited by J. R. Kubicki (John Wiley \& Sons, Ltd., 2016), pp. 107-149.

${ }^{271}$ J. C. Phillips, Phys. Rev. 112, 685 (1958)

${ }^{272}$ J. C. Phillips and L. Kleinman, Phys. Rev. 116, 287 (1959).

${ }^{273}$ B. J. Austin, V. Heine, and L. J. Sham, Phys. Rev. 127, 276 (1962).

${ }^{274}$ M. T. Yin and M. L. Cohen, Phys. Rev. B 25, 7403 (1982).

${ }^{275}$ N. A. W. Holzwarth, G. E. Matthews, R. B. Dunning, A. R. Tackett, and Y. Zeng, Phys. Rev. B 55, 2005 (1997).

${ }^{276}$ G. Kresse and D. Joubert, Phys. Rev. B 59, 1758 (1999).

${ }^{277}$ M. Valiev and J. H. Weare, J. Phys. Chem. A 103, 10588 (1999).

${ }^{278}$ R. Atta-Fynn, E. J. Bylaska, and W. A. De Jong, MRS Online Proc. Libr. Archiv. 1383, mrsf11-1383-a07-06 (2012).

${ }^{279}$ R. Atta-Fynn, E. J. Bylaska, and W. A. de Jong, J. Phys. Chem. Lett. 4, 2166 (2013).

${ }^{280}$ S. O. Odoh, E. J. Bylaska, and W. A. de Jong, J. Phys. Chem. A 117, 12256 (2013).

${ }^{281}$ R. Atta-Fynn, E. J. Bylaska, and W. A. de Jong, J. Phys. Chem. A 120, 10216 (2016).

${ }^{282}$ R. Atta-Fynn, E. J. Bylaska, and W. A. de Jong, "Finite temperature free energy calculations in NWChem: Metadynamics and umbrella sampling-WHAM," http://github.com/nwchemgit/nwchem/wiki/nwchem-new-pmf.pdf.

${ }^{283}$ D. Meng, B. Zheng, G. Lin, and M. L. Sushko, Commun. Comput. Phys. 16, 1298 (2014)

${ }^{284}$ D. Osei-Kuffuor and J.-L. Fattebert, Phys. Rev. Lett. 112, 046401 (2014).

${ }^{285}$ A. Klamt and G. Schurmann, J. Chem. Soc., Perkin Trans. 2 1993, 799.

${ }^{286}$ A. V. Marenich, C. J. Cramer, and D. G. Truhlar, J. Phys. Chem. B 113, 6378 (2009).

${ }^{287}$ G. M. Chaban, J. O. Jung, and R. B. Gerber, J. Chem. Phys. 111, 1823 (1999).

${ }^{288}$ Y.-Y. Chuang, D. G. Truhlar, R. A. Kendall, B. C. Garrett, and T. L. Windus, "DIRDYVTST," https://github.com/nwchemgit/nwchem/wiki/Interface, 2004, online; accessed 15 February 2020.

${ }^{289}$ J. Zheng, J. L. Bao, R. Meana-Pañeda, S. Zhang, B. J. Lynch, J. C. Corchado, Y.Y. Chuang, P. L. Fast, W.-P. Hu, Y.-P. Liu, G. C. Lynch, K. A. Nguyen, C. F. Jackels, A. Fernandez Ramos, B. A. Ellingson, V. S. Melissas, J. Villà, I. Rossi, E. L. Coitiño, J. Pu, T. V. Albu, A. Ratkiewicz, R. Steckler, B. C. Garrett, A. D. Isaacson, and
D. G. Truhlar, "Polyrate 17-C: Computer program for the calculation of chemical reaction rates for polyatomics," https://comp.chem.umn.edu/polyrate, 2017, online; accessed 15 February 2020.

${ }^{290}$ See https://wiki.fysik.dtu.dk/ase/ for Atomic Simulation Environment.

${ }^{291}$ E. D. Hermes, K. Sargsyan, H. N. Najm, and J. Zádor, J. Chem. Theory Comput. 15, 6536 (2019).

${ }^{292}$ See https://github.com/zadorlab/sella for Sella: A utility for finding first order saddle points.

${ }^{293}$ E. J. Bylaska, J. Q. Weare, and J. H. Weare, J. Chem. Phys. 139, 074114 (2013).

${ }^{294} \mathrm{H}$. Jónsson, G. Mills, and K. Jacobsen, "Nudged elastic band method for finding minimum energy paths of transitions," in Classical and Quantum Dynamics in Condensed Phase Simulations, edited by B. J. Berne, G. Ciccotti, and D. F. Coker (World Scientific, 1998), pp. 385-404.

${ }^{295}$ G. Henkelman and H. Jónsson, J. Chem. Phys. 113, 9978 (2000).

${ }^{296} \mathrm{G}$. Henkelman, B. P. Uberuaga, and H. Jónsson, J. Chem. Phys. 113, 9901 (2000).

${ }^{297}$ S. Smidstrup, A. Pedersen, K. Stokbro, and H. Jónsson, J. Chem. Phys. 140, 214106 (2014).

${ }^{298}$ W. E, W. Ren, and E. Vanden-Eijnden, J. Chem. Phys. 126, 164103 (2007).

${ }^{299}$ V. E. Henson, in Computational Imaging, Vol. 5016, edited by C. A. Bouman and R. L. Stevenson (SPIE, 2003), pp. 36-48.

${ }^{300}$ E. J. Bylaska, "Python-NEB," https://bitbucket.org/ebylaska/python-neb.

${ }^{301}$ T. P. Straatsma and J. A. McCammon, J. Comput. Chem. 11, 943 (1990).

${ }^{302}$ T. P. Straatsma, M. Philippopoulos, and J. A. McCammon, Comput. Phys. Commun. 128, 377 (2000).

${ }^{303}$ T. P. Straatsma, J. Phys.: Conf. Ser. 16, 287 (2005).

${ }^{304}$ T. P. Straatsma and D. G. Chavarría-Miranda, Comput. Phys. Commun. 184, 2634 (2013).

${ }^{305}$ T. P. Straatsma and V. Helms, Molecular Dynamics Module of NWChem Design and Application in Protein Simulations (World Scientific Pub. Co., 2000), pp. $70-82$.

${ }^{306}$ W. Gu, T. Frigato, T. P. Straatsma, and V. Helms, Angew. Chem., Int. Ed. 46, 2939 (2007).

${ }^{307}$ R. D. Lins and T. P. Straatsma, Biophys. J. 81, 1037 (2001).

${ }^{308}$ R. M. Shroll and T. P. Straatsma, Biopolymers 65, 395 (2002).

${ }^{309}$ T. P. Straatsma, in Abstracts of Papers of the American Chemical Society (American Chemical Society, 2005), Vol. 230, p. U1295.

${ }^{310}$ T. A. Soares and T. P. Straatsma, Mol. Simul. 34(3), 295 (2008).

${ }^{311}$ T. A. Soares, T. P. Straatsma, and R. D. Lins, J. Braz. Chem. Soc. 19(2), 312 (2008).

${ }^{312}$ R. D. Lins, E. R. Vorpagel, M. Guglielmi, and T. P. Straatsma, Biomacromolecules 9, 29 (2008).

${ }^{313}$ R. M. Shroll and T. P. Straatsma, Mol. Simul. 29, 1 (2003).

${ }^{314}$ R. M. Shroll and T. P. Straatsma, Biophys. J. 84, 1765 (2003).

${ }^{315}$ T. P. Straatsma, in Abstracts of Paper of the American Chemical Society (American Chemical Society, 2003), Vol. 225, p. U805.

${ }^{316}$ A. R. Felmy, C. Liu, and T. P. Straatsma, Geochim. Cosmochim. Acta 69(10), A171 (2005).

${ }^{317}$ T. P. Straatsma, "Lipopolysaccharide membranes and membrane proteins of Pseudomonas aeruginosa studied by computer simulation," in Recent Progress in Computational Sciences and Engineering, Lecture Series on Computer and Computational Sciences (VSP Brill, 2006), Vol. 7B, pp. 519-525.

${ }^{318}$ T. A. Soares and T. P. Straatsma, AIP Conf. Proc. 963, 1375 (2007).

${ }^{319}$ T. P. Straatsma and T. A. Soares, Proteins: Struct., Funct., Bioinf. 74, 475 (2009).

${ }^{320}$ U. Essmann, L. Perera, M. L. Berkowitz, T. Darden, H. Lee, and L. G. Pedersen, J. Chem. Phys. 103, 8577 (1995).

${ }^{321}$ J. W. Ponder and D. A. Case, "Protein simulations," Advances in Protein Chemistry Vol. 66 (Academic Press, 2003), pp. 27-85.

${ }^{322}$ K. Vanommeslaeghe, E. Hatcher, C. Acharya, S. Kundu, S. Zhong, J. E. Shim, E. Darian, O. Guvench, P. Lopes, I. Vorobyov, and J. A. MacKerell, J. Comput. Chem. 31, 671 (2010). 
${ }^{323}$ C. I. Bayly, P. Cieplak, W. Cornell, and P. A. Kollman, J. Phys. Chem. 97, 10269 (1993).

${ }^{324}$ W. D. Cornell, P. Cieplak, C. I. Bayly, and P. A. Kollman, J. Am. Chem. Soc. 115, 9620 (1993).

${ }^{325}$ T. P. Straatsma and J. A. McCammon, J. Chem. Phys. 95, 1175 (1991).

${ }^{326}$ T. P. Straatsma and J. A. McCammon, Methods Enzymol. 202, 497 (1991).

${ }^{327}$ T. P. Straatsma and J. A. McCammon, Annu. Rev. Phys. Chem. 43, 407 (1992).

${ }^{328}$ T. P. Straatsma, M. Zacharias, and J. A. McCammon, "Free energy difference calculations in biomolecular systems," in Computer Simulation of Biomolecular Systems, edited by W. F. van Gunsteren et al. (ESCOM, Leiden, 1993), pp. 349-367. ${ }^{329}$ T. P. Straatsma, "Free energy by molecular simulation," in Reviews in Computational Chemistry, edited by K. B. Lipkowitz and D. B. Boyd (John Wiley \& Sons, Ltd., 2007), Vol. IX, pp. 81-127.

${ }^{330}$ T. P. Straatsma, "Free energy simulations," in Encyclopedia of Computational Chemistry, edited by P. von Rague Schleyer (American Cancer Society, 2002), pp. 1083-1089.

${ }^{331}$ T. P. Straatsma, H. J. C. Berendsen, and A. J. Stam, Mol. Phys. 57, 89 (1986).

${ }^{332}$ M. Zacharias, T. P. Straatsma, and J. A. McCammon, J. Chem. Phys. 100, 9025 (1994).

${ }^{333}$ T. P. Straatsma, AIP Conf. Proc. 963, 1379 (2007).

${ }^{334}$ E. S. Peterson, E. G. Stephan, A. L. Corrigan, R. D. Lins, T. A. Soares, R. E. Scarberry, L. K. Williams, S. J. Rose, C. Lai, T. J. Critchlow, and T. P. Straatsma, in Proceedings of the International Conference on Bioinformatics and Computational Biology, Las Vegas, NV, July 14-17, 2008.

${ }^{335}$ T. P. Straatsma and J. A. McCammon, IBM Syst. J. 40, 328 (2001).

${ }^{336}$ A. V. Marenich, C. J. Cramer, D. G. Truhlar, C. A. Guido, B. Mennucci, G. Scalmani, and M. J. Frisch, Chem. Sci. 2, 2143 (2011).

${ }^{337}$ M. Valiev, B. C. Garrett, M.-K. Tsai, K. Kowalski, S. M. Kathmann, G. K. Schenter, and M. Dupuis, J. Chem. Phys. 127, 051102 (2007).

${ }^{338}$ M. Valiev, E. J. Bylaska, M. Dupuis, and P. G. Tratnyek, J. Phys. Chem. A 112, 2713 (2008).

${ }^{339}$ D. Case, I. Ben-Shalom, S. Brozell, D. Cerutti, T. Cheatham III, V. Cruzeiro, R. D. T. A. Darden, D. Ghoreishi, G. Giambasu, T. Giese, M. Gilson, H. Gohlke, A. Goetz, N. H. D. Greene, R. Harris, Y. Huang, S. Izadi, A. Kovalenko, R. Krasny, T. Kurtzman, T. Lee, S. LeGrand, P. Li, J. L. C. Lin, T. Luchko, R. Luo, V. Man, D. Mermelstein, K. Merz, Y. Miao, G. Monard, C. Nguyen, H. Nguyen, A. Onufriev, F. Pan, R. Qi, D. Roe, A. Roitberg, C. Sagui, S. Schott-Verdugo, J. Shen, C. L. Simmerling, J. Smith, J. Swails, R. Walker, J. Wang, H. Wei, L. Wilson, R. Wolf, X. Wu, L. Xiao, Y. Xiong, D. York, and P. Kollman, AMBER 2019 (University of California, San Francisco, 2019).

${ }^{340}$ T. A. Soares, M. A. Osman, and T. P. Straatsma, J. Chem. Theory Comput. 3, 1569 (2007).

${ }^{341}$ D. E. B. Gomes, R. D. Lins, P. G. Pascutti, C. Lei, and T. A. Soares, J. Phys. Chem. B 115, 15389 (2011).

${ }^{342}$ D. E. B. Gomes, R. D. Lins, P. G. Pascutti, C. Lei, and T. A. Soares, J. Phys. Chem. B 114, 531 (2010).

${ }^{343}$ B. H. Lower, R. D. Lins, Z. Oestreicher, T. P. Straatsma, M. F. Hochella, L. Shi, and S. K. Lower, Environ. Sci. Technol. 42, 3821 (2008).

${ }^{344}$ J.-F. Boily and R. D. Lins, J. Phys. Chem. C 113, 16568 (2009).

${ }^{345}$ I. Chandrasekhar, M. Kastenholz, R. D. Lins, C. Oostenbrink, L. D. Schuler, D. P. Tieleman, and W. F. van Gunsteren, Eur. Biophys. J. 32, 67 (2003).

${ }^{346}$ R. D. Lins and P. H. Hünenberger, J. Comput. Chem. 26, 1400 (2005).

${ }^{347}$ L. Pol-Fachin, V. H. Rusu, H. Verli, and R. D. Lins, J. Chem. Theory Comput. 8, 4681 (2012).

${ }^{348}$ L. Pol-Fachin, H. Verli, and R. D. Lins, J. Comput. Chem. 35, 2087 (2014).

${ }^{349}$ T. A. Soares, P. H. Hünenberger, M. A. Kastenholz, V. Kräutler, T. Lenz, R. D. Lins, C. Oostenbrink, and W. F. van Gunsteren, J. Comput. Chem. 26, 725 (2005).

${ }^{350}$ E. F. Franca, L. C. G. Freitas, and R. D. Lins, Biopolymers 95, 448 (2011).

${ }^{351}$ E. F. Franca, R. D. Lins, L. C. G. Freitas, and T. P. Straatsma, J. Chem. Theory Comput. 4, 2141 (2008).
${ }^{352}$ M. Svensson, S. Humbel, R. D. J. Froese, T. Matsubara, S. Sieber, and K. Morokuma, J. Phys. Chem. 100, 19357 (1996).

${ }^{353}$ G. N. Chuev, M. V. Fedotova, and M. Valiev, J. Chem. Phys. 152, 041101 (2020).

${ }^{354}$ M. Valiev and G. N. Chuev, J. Stat. Mech.: Theory Exp. 2018, 093201.

${ }^{355}$ G. N. Chuev, M. Valiev, and M. V. Fedotova, J. Chem. Theory Comput. 8, 1246 (2012).

${ }^{356}$ D. Chandler, J. D. McCoy, and S. J. Singer, J. Chem. Phys. 85, 5971 (1986).

${ }^{357}$ D. Chandler, J. D. McCoy, and S. J. Singer, J. Chem. Phys. 85, 5977 (1986).

${ }^{358}$ J. D. Owens, D. Luebke, N. Govindaraju, M. Harris, J. Krüger, A. E. Lefohn, and T. J. Purcell, Comput. Graphics Forum 26, 80 (2007).

${ }^{359} \mathrm{~N}$. Gawande, K. Kowalski, B. Palmer, S. Krishnamoorthy, E. Apra, J. Manzano, V. Amatya, and J. Crawford, in 2019 IEEE/ACM Workshop on Exascale MPI (ExaMPI) (IEEE, 2019), pp. 29-38.

${ }^{360}$ C. Yu, W. Harbich, L. Sementa, L. Ghiringhelli, E. Aprá, M. Stener, A. Fortunelli, and H. Brune, J. Chem. Phys. 147, 074301 (2017).

${ }^{361}$ J. R. Hammond, K. Kowalski, and W. A. deJong, J. Chem. Phys. 127, 144105 (2007).

${ }^{362}$ B. Peng, N. Govind, E. Aprà, M. Klemm, J. R. Hammond, and K. Kowalski, J. Phys. Chem. A 121, 1328 (2017).

${ }^{363}$ H.-S. Hu, K. Bhaskaran-Nair, E. Aprà, N. Govind, and K. Kowalski, J. Phys. Chem. A 118, 9087 (2014).

${ }^{364}$ W. A. de Jong, E. Bylaska, N. Govind, C. L. Janssen, K. Kowalski, T. Müller, I. M. B. Nielsen, H. J. J. van Dam, V. Veryazov, and R. Lindh, Phys. Chem. Chem. Phys. 12, 6896 (2010).

${ }^{365}$ J. S. Nelson, S. J. Plimpton, and M. P. Sears, Phys. Rev. B 47, 1765 (1993).

${ }^{366}$ J. Wiggs and H. Jónsson, Comput. Phys. Commun. 87, 319 (1995).

${ }^{367}$ A. Canning and D. Raczkowski, Comput. Phys. Commun. 169, 449 (2005).

${ }^{368}$ F. Gygi, IBM J. Res. Dev. 52, 137 (2008).

${ }^{369}$ T. W. Swaddle, J. Rosenqvist, P. Yu, E. J. Bylaska, B. L. Phillips, and W. H. Casey, Science 308, 1450 (2005).

${ }^{370}$ J. R. Rustad and E. J. Bylaska, J. Am. Chem. Soc. 129, 2222 (2007).

${ }^{371}$ R. Atta-Fynn, D. F. Johnson, E. J. Bylaska, E. S. Ilton, G. K. Schenter, and W. A. de Jong, Inorg. Chem. 51, 3016 (2012).

${ }^{372}$ J. L. Fulton, E. J. Bylaska, S. Bogatko, M. Balasubramanian, E. Cauët, G. K. Schenter, and J. H. Weare, J. Phys. Chem. Lett. 3, 2588 (2012).

${ }^{373}$ M. D. Hanwell, D. E. Curtis, D. C. Lonie, T. Vandermeersch, E. Zurek, and G. R. Hutchison, J. Cheminf. 4, 17 (2012).

${ }^{374}$ See http://www.jmol.org/ for Jmol: An open-source Java viewer for chemical structures in 3D.

${ }^{375}$ See https://www.chemcraftprog.com for Chemcraft-Graphical software for visualization of quantum chemistry computations.

${ }^{376}$ See http://mocalc2012.sourceforge.net/ for Mocalc2012.

${ }^{377}$ N. M. O’boyle, A. L. Tenderholt, and K. M. Langner, J. Comput. Chem. 29, 839 (2008).

${ }^{378}$ See http://www.chemissian.com/ for Chemissian.

${ }^{379}$ See http://www.scienomics.com for Scienomics, MAPS Platform.

${ }^{380}$ See http://www.biomolecular-modeling.com/Ascalaph/ for Ascalaph.

${ }^{381}$ G. Black, K. Schuchardt, D. Gracio, and B. Palmer, in Computational ScienceICCS 2003, edited by P. M. A. Sloot, D. Abramson, A. V. Bogdanov, Y. E. Gorbachev, J. J. Dongarra, and A. Y. Zomaya (Springer Berlin Heidelberg, Berlin, Heidelberg, 2003), pp. 122-131.

${ }^{382}$ See http://www-stone.ch.cam.ac.uk/programs.html for CamCASP (Cambridge package for Calculation of Anisotropic Site Properties).

${ }^{383}$ See http://www.chemshell.org for ChemShell: A Computational Chemistry Shell.

${ }^{384}$ T. Y. Nikolaienko, L. A. Bulavin, and D. M. Hovorun, Comput. Theor. Chem. 1050, 15 (2014).

${ }^{385}$ S. Canneaux, F. Bohr, and E. Henon, J. Comput. Chem. 35, 82 (2014).

${ }^{386}$ U. Lourderaj, R. Sun, S. C. Kohale, G. L. Barnes, W. A. de Jong, T. L. Windus, and W. L. Hase, Comput. Phys. Commun. 185, 1074 (2014).

${ }^{387}$ See http://perso.neel.cnrs.fr/xavier.blase/fiesta/index.html for Fiesta. 
${ }^{388}$ See https://pupil.sourceforge.net for PUPIL-Program for User Package Interface and Linking.

${ }^{389}$ J. Wehner, L. Brombacher, J. Brown, C. Junghans, O. Çaylak, Y. Khalak, P. Madhikar, G. Tirimbò, and B. Baumeier, J. Chem. Theory Comput. 14, 6253 (2018).

${ }^{390}$ See https://github.com/KristapsE/PyDP4 for Pydp4.

${ }^{391}$ A. Supady, V. Blum, and C. Baldauf, J. Chem. Inf. Model. 55, 2338 (2015).

${ }^{392}$ See https://gaussian.com/cubegen/ for Gaussian cube.

${ }^{393}$ G. Schaftenaar, E. Vlieg, and G. Vriend, J. Comput.-Aided Mol. Des. 31, 789 (2017).

${ }^{394}$ See https://ugovaretto.github.io/molekel/ for Molekel 5.4.

${ }^{395}$ D. L. Bergman, L. Laaksonen, and A. Laaksonen, J. Mol. Graphics Modell. 15, 301 (1997).

${ }^{396}$ W. Humphrey, A. Dalke, and K. Schulten, J. Mol. Graphics 14, 33 (1996).

${ }^{397}$ See https://sourceforge.net/projects/jbonzer/ for Jamberoo.

${ }^{398} \mathrm{~K}$. Momma and F. Izumi, J. Appl. Crystallogr. 41, 653 (2008).

${ }^{399}$ J. Contreras-García, E. R. Johnson, S. Keinan, R. Chaudret, J.-P. Piquemal,

D. N. Beratan, and W. Yang, J. Chem. Theory Comput. 7, 625 (2011).

${ }^{400}$ See http://www.quimica.urv.es/XAIM for Xaim.

${ }^{401} \mathrm{~T}$. Lu and F. Chen, J. Comput. Chem. 33, 580 (2012).

${ }^{402}$ S. Ghosh, "SEMIEMP: Open source code for semiempirical qunatum chemistry calculation," http://github.com/SoumenChem/semiemp.

${ }^{403}$ S. Ghosh, A. Andersen, L. Gagliardi, C. J. Cramer, and N. Govind, J. Chem. Theory Comput. 13, 4410 (2017).

${ }^{404}$ S. Ghosh, J. C. Asher, L. Gagliardi, C. J. Cramer, and N. Govind, J. Chem. Phys. 150, 104103 (2019).

${ }^{405}$ D. E. Bernholdt, B. A. Allan, R. Armstrong, F. Bertrand, K. Chiu, T. L. Dahlgren, K. Damevski, W. R. Elwasif, T. G. W. Epperly, M. Govindaraju, D. S. Katz, J. A. Kohl, M. Krishnan, G. Kumfert, J. W. Larson, S. Lefantzi, M. J. Lewis, A. D. Malony, L. C. Mclnnes, J. Nieplocha, B. Norris, S. G. Parker, J. Ray, S. Shende, T. L. Windus, and S. Zhou, Int. J. High Perform. Comput. Appl. 20, 163 (2006).

${ }^{406}$ R. M. Richard, C. Bertoni, J. S. Boschen, K. Keipert, B. Pritchard, E. F. Valeev, R. J. Harrison, W. A. de Jong, and T. L. Windus, Comput. Sci. Eng. 21, 48 (2019). ${ }^{407}$ J. P. Kenny, S. J. Benson, Y. Alexeev, J. Sarich, C. L. Janssen, L. C. Mcinnes, M. Krishnan, J. Nieplocha, E. Jurrus, C. Fahlstrom, and T. L. Windus, J. Comput. Chem. 25, 1717 (2004).
${ }^{408}$ T. P. Gulabani, M. Sosonkina, M. S. Gordon, C. L. Janssen, J. P. Kenny, H. Netzloff, and T. L. Windus, in Proceedings of the 2009 Spring Simulation Multiconference, SpringSim'09 (Society for Computer Simulation International, San Diego, CA, USA, 2009).

${ }^{409}$ M. Krishnan, Y. Alexeev, T. L. Windus, and J. Nieplocha, in SC'05: Proceedings of the 2005 ACM/IEEE Conference on Supercomputing (IEEE, 2005), p. 23.

${ }^{410}$ J. P. Kenny, C. L. Janssen, E. F. Valeev, and T. L. Windus, J. Comput. Chem. 29, 562 (2008).

${ }^{411}$ E. Mutlu, K. Kowalski, and S. Krishnamoorthy, in Proceedings of the 6th ACM SIGPLAN International Workshop on Libraries, Languages and Compilers for Array Programming (ACM, 2019), pp. 46-56.

${ }^{412}$ N. P. Bauman, E. J. Bylaska, S. Krishnamoorthy, G. H. Low, N. Wiebe, C. E. Granade, M. Roetteler, M. Troyer, and K. Kowalski, J. Chem. Phys. 151, 014107 (2019).

${ }^{413}$ J. Boström, M. Pitoňák, F. Aquilante, P. Neogrády, T. B. Pedersen, and R. Lindh, J. Chem. Theory Comput. 8, 1921 (2012).

${ }^{414}$ T. B. Pedersen, A. M. J. Sánchez de Merás, and H. Koch, J. Chem. Phys. 120, 8887 (2004).

${ }^{415}$ E. Epifanovsky, D. Zuev, X. Feng, K. Khistyaev, Y. Shao, and A. I. Krylov, J. Chem. Phys. 139, 134105 (2013).

${ }^{416}$ X. Feng, E. Epifanovsky, J. Gauss, and A. I. Krylov, J. Chem. Phys. 151, 014110 (2019).

${ }^{417}$ C. Peng, J. A. Calvin, and E. F. Valeev, Int. J. Quantum Chem. 119, e25894 (2019).

${ }^{418}$ S. D. Folkestad, E. F. Kjønstad, and H. Koch, J. Chem. Phys. 150, 194112 (2019).

${ }^{419}$ C. Riplinger and F. Neese, J. Chem. Phys. 138, 034106 (2013).

${ }^{420}$ C. Riplinger, P. Pinski, U. Becker, E. F. Valeev, and F. Neese, J. Chem. Phys. 144, 024109 (2016).

${ }^{421}$ F. Pavošević, C. Peng, P. Pinski, C. Riplinger, F. Neese, and E. F. Valeev, J. Chem. Phys. 146, 174108 (2017).

${ }^{422}$ G. Onida, L. Reining, and A. Rubio, Rev. Mod. Phys. 74, 601 (2002).

${ }^{423}$ B. Peng and K. Kowalski, J. Chem. Theory Comput. 14, 4335 (2018).

${ }^{424}$ G. H. Low, N. P. Bauman, C. E. Granade, B. Peng, N. Wiebe, E. J. Bylaska, D. Wecker, S. Krishnamoorthy, M. Roetteler, K. Kowalski, M. Troyer, and N. A. Baker, arXiv:1904.01131 (2019). 